M. SCHÖNHOFEN, M. CUBERO, B.L. FRIMAN,

W. NÖRENBERG, Gy. WOLF

Gesellschaft für Schwerionenforschung $\mathrm{mbH}$ Postfach 110552 - D-64220 Darmstadt · Germany 



\title{
Covariant Kinetic Equations and Relaxation Processes in Relativistic Heavy-Ion Collisions *
}

\author{
M. Schönhofen ${ }^{a}$, M. Cubero ${ }^{a}$, B.L. Friman ${ }^{a, b}$, \\ W. Nörenberg ${ }^{a, b}$ and Gy. Wolf ${ }^{a}$ \\ ${ }^{a}$ Gesellschaft für Schwerionenforschung (GSI) \\ Postfach 110552, D-64220 Darmstadt, Germany \\ ${ }^{b}$ Institut für Kernphysik, TH Darmstadt \\ Schloßgartenstraße 9, D-64289 Darmstadt, Germany
}

February 4, 1994

\begin{abstract}
We present a derivation of covariant kinetic equations for nucleons and deltas within a model of quantum hadrodynamics, which includes scalar, vector and pseudo-scalar mesons. By generalizing the Kadanoff-Baym equations to relativistic fields and using the gradient expansion as well as the quasiparticle approximation for the baryons, we find kinetic equations with covariant collision integrals of the Uehling-Uhlenbeck type. These relativistic Landau-Vlasov equations for nucleons and deltas are solved numerically for two counter-streaming nuclear-matter currents. Using the microscopic expressions we find - due to modifications of the baryon properties in nuclear matter - strong medium effects on relaxation processes in intermediate-energy heavy-ion collisions.
\end{abstract}

* Dedicated to Professor Klaus Dietrich on the occasion of his 60 th birthday 


\section{Introduction and summary}

Over the last decade relativistic heavy-ion collisions have been extensively investigated in order to gain information on nuclear matter at high densities and temperatures. A main objective is to determine the nuclear equation of state, i.e., the thermodynamical properties of nuclear matter, e.g. the pressure as function of temperature and baryon density. However, it is questionable whether thermodynamical equilibrium is attained in transient states such as those formed in relativistic heavy-ion collisions. Therefore, a consistent and reliable theoretical treatment must describe the complete dynamical evolution of nucleus-nucleus collisions.

Present theoretical descriptions of relativistic heavy-ion collisions are rather crude. The main activities have been concentrated on phase-space simulations using phenomenological collision integrals. The development of these approaches, i.e. cascade models [1] and Boltzmann-Uehling-Uhlenbeck transport theories [210], has been stimulated by their success in reproducing available experimental data. However, problems related to relativistic dynamics and to medium effects in relativistic heavy-ion collisions have not been studied systematically. The purpose of our paper is therefore threefold:

1. to present a comprehensive derivation of covariant kinetic equations (relativistic Landau-Vlasov equations) including the description of all inevitable approximations (cf. section 2),

2. to illustrate the medium effects on relaxation phenomena in a simple model (two counter-streaming nuclear-matter currents) which can be treated numerically, to correlate them with the stiffness of the nuclear equation of state (cf. section 3 ), and thereby

3. to establish a basis for further approximations leading e.g. to more macroscopic descriptions, like the relativistic multi-fluid models [11], which allow numerical solutions for realistic heavy-ion collisions (cf. the discussion in section 4).

A rigorous covariant description of strongly interacting many-particle systems is possible within quantum field theories, where the interacting meson and baryon fields are treated explicitly. The use of hadronic degrees of freedom rather than quarks and gluons is justified for laboratory energies $E_{\text {lab }} \leq 2 \mathrm{GeV} / \mathrm{u}$, cf. ref. [12]. Such theories are referred to as quantum hadrodynamics (QHD) [13]. In the simplest version of QHD one considers two isoscalar meson fields, i.e. a Lorentzscalar field $\Phi$ and a Lorentz-vector field $\omega^{\mu}$, which interact with the nucleon field $\Psi_{N}$. In the present work we use a generalization of this model, which includes the isovector-pseudoscalar pion field $\vec{\pi}$ and the delta field $\Psi_{\Delta}^{\mu}$. The lagrangian and the corresponding field equations are described in subsect. 2.1 .

Several authors have derived covariant kinetic equations for nuclear matter based on similar models. Elze et al. [14] have obtained a covariant mean-field 
equation without collision term for nucleons interacting via $\sigma$ - and $\omega$-mesons. This equation has been solved numerically by several groups [7-9] with the goal of eventually describing heavy-ion collisions. Ko et al. [8] have derived a covariant kinetic equation with a medium-dependent collision term for a system of nucleons interacting via $\sigma$ - and $\omega$-mesons using the non-equilibrium Green-function formalism of Kadanoff and Baym. However, in the numerical application they l.ave simplified the kinetic equation by introducing a phenomenological mediumindependent parametrization of the cross section in the collision term $[8,9]$. Recently, Mrówczyński and Heinz [15] have presented a detailed study of the transport equations for the Walecka model, which is similar to ours. They have gone beyond our considerations by taking spin degrees of freedom explicitly into account. However, their study does not include the delta resonance, and they have not attempted to find solutions to their kinetic equations.

In section 2 we present the derivation of covariant kinetic equations for baryons within quantum hadrodynamics. The starting point is the covariant formulation of the equations of motion for non-equilibrium Green functions on a real time contour following the non-relativistic formalism developed by Kadanoff and Baym [16] as well as the work of Martin and Schwinger [17] and Keldysh [18]. For a comprehensive and systematic study of non-relativistic Green functions on a time contour we refer the reader to refs. [19, 20].

In subsect. 2.2 we discuss the Kadanoff-Baym formalism, generalized to the relativistic baryonic fields of nucleons and deltas. The resulting equations of motion for the Green functions are far too complicated for a straightforward numerical treatment of nucleus-nucleus collisions. Therefore, in subsect. 2.3, we carry out a gradient expansion in order to reduce the complexity of the coupled set of equations. Performing a Fourier transformation with respect to the relative coordinates one obtains equations of motion for the Green functions in the phase-space representation (Wigner transforms). These equations are further simplified by introducing the quasi-particle approximation for the baryons, which implies that the quasi-particles are assumed to propagate on mass shell between collisions. Hence, the original eight-dimensional phase space is reduced to a seven-dimensional hypersurface. The quasi-particle approximation is reliable, when the mean free path of the scattering particles is large compared to the de Broglie wave length of the particles.

The final form of the covariant kinetic equations (relativistic Landau-Vlasov equations) for the phase-space densities of the nucleons contain two different contributions from the nucleon-meson interaction: the mean fields and the collision term. The matrix elements for the scattering amplitudes in the collision integrals are calculated in Born approximation with mean-field spinors (cf. subsect. 2.4) Already at this level of the perturbation expansion, the calculation of the scattering amplitudes is quite involved. Thus, the inclusion of higher-order terms does not seem to be practicable. To some extent we compensate for the short-comings of the Born approximation by fitting the coupling constants and form factors to the nucleon-nucleon scattering cross sections. A drawback of the Born approxima- 
tion is that the corresponding scattering amplitude is not unitary. We shall not address this problem here, but note that to cure this problem a $\mathrm{T}$ - or $\mathrm{K}$-matrix approach would be needed. In subsect. 2.5 the technical details and extensions are discussed, which are needed in the derivation of the kinetic equation for the deltas (spin $=\frac{3}{2}$ and isospin $=\frac{3}{2}$ ). Thus we finally obtain kinetic equations which allow us to study medium effects on the equilibration processes in intermediate-energy heavy-ion collisions.

The numerical treatment of nucleus-nucleus collisions in a kinetic model, such as the one developed here, is quite complicated, because it requires the solution of an initial-value problem in $6+1$ dimensions (coordinate space, momentum space and time). Therefore, we explore the relaxation process in the infinite system of two counter-streaming nuclear-matter currents (cf. section 3). For such a model the kinetic equations can be treated numerically and the relevance of medium effects in the relaxation process can be studied.

In subsect. 3.1 we determine the model parameters by fitting the elastic differential nucleon-nucleon cross section at $E_{\mathrm{lab}}=1 \mathrm{GeV}$. The coupling constant and the form factor of the pion-nucleon-delta vertex are taken from refs. [21, 22]. The dependence on angle and energy of the elastic and inelastic cross sections agrees quite well with experimental data.

In subsect. 3.2 we solve the coupled set of covariant kinetic equations for the baryons in the system of two counter-streaming nuclear-matter currents, where the initial distance of the two Fermi ellipsoids corresponds to an incident energy of $E_{\mathrm{lab}}=1 \mathrm{GeV} / \mathrm{u}$. In order to study the medium effects in some detail we have performed three different kinds of calculations: one without mean-field contributions and two including mean-field effects with different sets of coupling constants. A major effect, which influences the equilibration process, is a fairly large shift of the initial momentum distributions towards each other due to the strong mean fields. Furthermore, the quasi-particle masses of the baryons are reduced below their vacuum values. This reduction of the effective masses in medium leads to enhanced elastic and inelastic nucleon-nucleon cross sections and modifies the angular dependence of the differential cross sections. As a result of both effects, we find strong reductions of the equilibration times as compared to calculations, where these medium effects are not taken into account.

The relativistic Landau-Vlasov equations cannot be solved numerically for realistic heavy-ion collisions, because the evaluation of the collision terms becomes too involved. It is, however, a good basis for further approximations, yielding e.g. two- or three-fluid models which contain the essential effects from mesonic fields in the mean-field part and in the collision term [11]. Moreover, our results for two counter-streaming nuclear-matter currents can be used for testing such new developments (cf. section 4). 


\section{Covariant kinetic equations}

In this section we derive covariant kinetic equations for the baryons within a quantum hadrodynamical model which describes the interacting system of nucleons, deltas, sigmas, omegas and pions (cf. subsect. 2.1). In subsects. $2.2-2.4$ we perform the individual steps which finally yield the relativistic Landau-Vlasov equations for nucleons. The extension to deltas is discussed in subsect. 2.5.

\subsection{Lagrangian and field equations}

In order to describe hot and dense hadronic matter, a consistent microscopic model for strongly interacting relativistic quantum-mechanical systems is needed. A suitable framework for such studies is provided by quantum hadrodynamics [13], a relativistic quantum field theory of mesons and baryons. The corresponding fields are listed in table 1 . The Lagrange density $\mathcal{L}$ is given by

$$
\begin{aligned}
\mathcal{L} & =\bar{\Psi}_{N}\left(\gamma_{\mu}\left(i \partial^{\mu}-g_{\omega} \omega^{\mu}\right)-m_{N}+g_{\sigma} \Phi\right) \Psi_{N} \\
& +\bar{\Psi}_{\Delta \nu}\left(\gamma_{\mu}\left(i \partial^{\mu}-g_{\omega} \omega^{\mu}\right)-m_{\Delta}+g_{\sigma} \Phi\right) \Psi_{\Delta}^{\nu} \\
& +\frac{1}{2}\left(\partial_{\mu} \Phi \partial^{\mu} \Phi-m_{\sigma}^{2} \Phi^{2}\right)-\frac{1}{3} b \Phi^{3}-\frac{1}{4} c \Phi^{4} \\
& -\frac{1}{4} F_{\mu \nu} F^{\mu \nu}+\frac{1}{2} m_{\omega}^{2} \omega_{\nu} \omega^{\nu} \\
& +\frac{1}{2}\left(\partial_{\mu} \vec{\pi} \partial^{\mu} \vec{\pi}-m_{\pi}^{2} \vec{\pi}^{2}\right) \\
& +i g_{\pi} \bar{\Psi}_{N} \vec{\tau} \gamma_{5} \Psi_{N} \vec{\pi} \\
& -g_{\pi N \Delta} \bar{\Psi}_{N} \vec{T} \Psi_{\Delta \nu} \partial^{\nu} \vec{\pi}-g_{\pi N \Delta} \bar{\Psi}_{\Delta \nu} \vec{T}^{\dagger} \Psi_{N} \partial^{\nu} \vec{\pi} .
\end{aligned}
$$

Here

$$
F_{\mu \nu}=\partial_{\mu} \omega_{\nu}-\partial_{\nu} \omega_{\mu}
$$

denotes the field-strength tensor of the $\omega$-meson, $g_{\sigma}, g_{\omega}$ and $g_{\pi}$ are the coupling constants of the nucleons to the scalar, vector and pseudo-scalar meson fields, respectively, and $g_{\pi N \Delta}$ is the coupling constant for the $\pi N \Delta$ interaction vertex. Whereas the nucleon field $\Psi_{N}$ is a four-component spinor field, the delta is represented by a vector-spinor field $\Psi_{\Delta}^{\nu}$ with twelve independent components. The subsidiary condition

$$
\gamma_{\nu} \Psi_{\Delta}^{\nu}=0
$$

leaves us finally with eight physical degrees of freedom which describe a spin- $\frac{3}{2}$ field [23]. We assume that the vector meson couples to the conserved baryon current. Thus, the coupling constants for the vector-meson interaction with nucleons and deltas are equal. Furthermore, we also use the same constants for the coupling of the scalar meson to nucleons and deltas. Thus, the difference of the effective masses is independent of the expectation value $\langle\Phi\rangle$ of the scalar field, and consequently of the density and temperature of nuclear matter. The baryon field operators $\Psi_{N}$ and $\Psi_{\Delta}^{\nu}$ satisfy the anti-commutation relations for fermions and the meson 
field operators $\Phi, \omega^{\mu}, \vec{\pi}$ the commutation relations for bosons. The components of the vector $\vec{\tau}$ are the Pauli-matrices in isospin space, and $\vec{T}$ denotes the isospin transition matrices which connect the isospin- $\frac{1}{2}$ and $-\frac{3}{2}$ states of the nucleon and the delta, respectively. A general discussion as well as an explicit representation of these matrices can be found e.g. in ref. [22].

By varying the action integral one obtains the following field equations:

$$
\begin{gathered}
\left\{\gamma^{\mu}\left(i \partial_{\mu}-g_{\omega} \omega_{\mu}\right)-m_{N}+g_{\sigma} \Phi+i g_{\pi} \gamma_{5} \vec{\tau} \vec{\pi}\right\} \Psi_{N}=g_{\pi N \Delta} \vec{T} \Psi_{\Delta \nu}\left(\partial^{\nu} \vec{\pi}\right) \\
\left\{\gamma^{\mu}\left(i \partial_{\mu}-g_{\omega} \omega_{\mu}\right)-m_{\Delta}+g_{\sigma} \Phi\right\} \Psi_{\Delta}^{\nu}=g_{\pi N \Delta} \vec{T}^{\dagger} \Psi_{N}\left(\partial^{\nu} \vec{\pi}\right) \\
\left(\partial_{\mu} \partial^{\mu}+m_{\pi}^{2}\right) \vec{\pi}= \\
\quad i g_{\pi} \bar{\Psi}_{N} \vec{\tau} \gamma_{5} \Psi_{N} \\
+g_{\pi N \Delta} \partial_{\mu} \bar{\Psi}_{N} \vec{T} \Psi_{\Delta}^{\mu}+g_{\pi N \Delta} \partial_{\mu} \bar{\Psi}_{\Delta}^{\mu} \vec{T}^{\dagger} \Psi_{N} \\
\partial_{\mu} F^{\mu \nu}+m_{\omega}^{2} \omega^{\nu}=g_{\omega} j_{B}^{\nu}, \\
\left(\partial_{\mu} \partial^{\mu}+m_{\sigma}^{2}\right) \Phi=g_{\sigma} \rho_{S},
\end{gathered}
$$

where the scalar density is given by

$$
\rho_{S}=\bar{\Psi}_{N} \Psi_{N}+\bar{\Psi}_{\Delta}^{\mu} \Psi_{\Delta \mu}
$$

and the baryon current by

$$
j_{B}^{\mu}=\bar{\Psi}_{N} \gamma^{\mu} \Psi_{N}+\bar{\Psi}_{\Delta}^{\nu} \gamma^{\mu} \Psi_{\Delta \nu}
$$

In the Hartree approximation the field operators $\Phi$ and $\omega^{\mu}$ of the scalar and vector mesons are replaced by their expectation values $\langle\Phi\rangle$ and $\left\langle\omega^{\mu}\right\rangle$. At this point it is convenient to introduce effective masses

$$
\begin{aligned}
& m_{N}^{*}=m_{N}-g_{\sigma}\langle\Phi\rangle \\
& m_{\Delta}^{*}=m_{\Delta}-g_{\sigma}\langle\Phi\rangle
\end{aligned}
$$

for the baryons.

\subsection{Covariant Kadanoff-Baym equations}

The original non-relativistic formulation of Kadanoff and Baym [16] is generalized to the QHD model in the following way. We consider the one-nucleon Green function

$$
\begin{aligned}
G\left(t_{1}, \boldsymbol{r}_{1}, \alpha_{1} ; t_{2}, \boldsymbol{r}_{2}, \alpha_{2}\right) & =G\left(x_{1}, \alpha_{1} ; x_{2}, \alpha_{2}\right)=G(1,2) \\
& =-i\left\langle T_{c}\left(\Psi_{H}(1) \bar{\Psi}_{H}(2)\right)\right\rangle
\end{aligned}
$$

for the nucleons, where $\alpha_{i}$ denote the spinor indices and $\langle\ldots\rangle$ the expectation value in a state of the interacting system at time $t_{0}$. For the moment this state remains 
unspecified. The operator $T_{c}$ is the causal time-ordering operator which orders an operator at an earlier time to the right of an operator at a later time. The field operators $\Psi_{H}(\mathrm{t})$ and $\bar{\Psi}_{H}(t)$ in the Heisenberg picture are given by

$$
\begin{aligned}
& \Psi_{H}(t)=\exp \left(i H\left(t-t_{0}\right)\right) \Psi \exp \left(-i H\left(t-t_{0}\right)\right), \\
& \bar{\Psi}_{H}(t)=\exp \left(i H\left(t-t_{0}\right)\right) \bar{\Psi} \exp \left(-i H\left(t-t_{0}\right)\right),
\end{aligned}
$$

where $H$ is the Hamiltonian of the system.

In addition to the causal Green function $G$, the one-nucleon correlation Green functions

$$
\begin{aligned}
& G^{<}(1,2)=+i\left\langle\bar{\Psi}_{H}(2) \Psi_{H}(1)\right\rangle \\
& G^{>}(1,2)=-i\left\langle\Psi_{H}(1) \bar{\Psi}_{H}(2)\right\rangle
\end{aligned}
$$

are needed in the description of systems in (equilibrium or non-equilibrium) excited states $[8,16]$.

The perturbation expansion is formulated in the interaction picture. We need the unitary time-evolution operator

$$
U\left(t, t_{0}\right)=T_{c}\left\{\exp \left(i \int_{t_{0}}^{t} d t^{\prime} \int d^{3} r \mathcal{L}_{I}^{1}\left(t^{\prime}, \mathbf{r}\right)\right)\right\},
$$

where $\mathcal{L}_{I}^{1}$ is the interaction part of the Lagrange density (in the interaction picture). An operator $O_{H}(t)$ in the Heisenberg picture is related to its counterpart in the interaction picture by

$$
\begin{aligned}
O_{H}(t)= & T_{a}\left\{\exp \left[i \int_{t}^{t_{0}} d t^{\prime} \int d^{3} r \mathcal{L}_{I}^{1}\left(t^{\prime}\right)\right]\right\} O_{I}(t) \\
& \cdot T_{c}\left\{\exp \left[i \int_{t_{0}}^{t} d t^{\prime} \int d^{3} r \mathcal{L}_{I}^{1}\left(t^{\prime}\right)\right]\right\}
\end{aligned}
$$

Here $T_{c}$ is again the causal time-ordering operator, whereas $T_{a}$ denotes the anticausal one which orders the operators at earlier times left to the ones at later times.

By introducing an integration contour in the complex time plane (see fig. 1), both types of time ordering can be conveniently subsumed into a time-ordering operator $T$ which orders the field operators according to the sequence of their time arguments on the contour $C[18,19]$. Thus, times on the causal (upper) branch of $C$ are earlier than times on the anti-causal (lower) branch. Using (16) we define the one-particle Green function (12) for time arguments on the contour $C$

$$
\begin{aligned}
i G(1,2) & =<T\left\{\Psi_{H}(1) \bar{\Psi}_{H}(2)\right\}> \\
& =<T\left\{\exp \left[i \int_{C} d t^{\prime} \int d^{3} r \mathcal{L}_{I}^{1}\left(t^{\prime}, \mathbf{r}\right)\right] \Psi_{I}(1) \bar{\Psi}_{I}(2)\right\}>,
\end{aligned}
$$

where the integration over $t^{\prime}$ is along $C$. The contour begins and ends at time $t_{0}$; the times $t_{1}$ and $t_{2}$ are located on the contour between the starting and end points. 
The retarded $\left(G^{+}\right)$and advanced $\left(G^{-}\right)$one-particle Green functions - generalized to the time contour - can be expressed in terms of the correlation functions (14) and the contour step function $\Theta_{C}$

$$
\begin{aligned}
& G^{+}(1,2)=\Theta_{C}\left(t_{1}-t_{2}\right)\left(G^{>}(1,2)-G^{<}(1,2)\right), \\
& G^{-}(1,2)=\Theta_{C}\left(t_{2}-t_{1}\right)\left(G^{<}(1,2)-G^{>}(1,2)\right) .
\end{aligned}
$$

The following relations between the Green functions exist:

$$
\begin{gathered}
G(1,2)=\Theta_{C}\left(t_{1}-t_{2}\right) G^{>}(1,2)+\Theta_{C}\left(t_{2}-t_{1}\right) G^{<}(1,2) \\
G^{+}(1,2)-G^{-}(1,2)=G^{>}(1,2)-G^{<}(1,2) \\
\left\{i G^{>}(1,2)\right\}^{\dagger}=i G^{>}(2,1) \\
\left\{i G^{<}(1,2)\right\}^{\dagger}=i G^{<}(2,1) \\
\left\{G^{+}(1,2)\right\}^{\dagger}=G^{-}(2,1) .
\end{gathered}
$$

The generalization of the two-particle Green function

$$
G_{2}(1,2 ; 3,4)=-i<T\left\{\Psi_{H}(1) \Psi_{H}(2) \bar{\Psi}_{H}(3) \bar{\Psi}_{H}(4)\right\}>
$$

to the time contour $C$ is completely analogous to the one-particle case. In fact, a Martin-Schwinger hierarchy [17] for contour-ordered Green functions can be formulated using the equations of motion for the field operators (eqs. (3) and (4)) as a starting point.

So far, the initial state at time $t_{0}$ has not been specified. This state contains all correlations which developed in the system at times prior to $t_{0}$. Such initial correlations are not considered here, because we shall take the limit $t_{0} \rightarrow-\infty$ where initial correlations may be neglected (adiabatic switching on of the interactions).

The series expansion of the exponential function in eq.(17) generates the perturbation expansion for one-particle Green function. In lowest order one obtains the non-interacting Green function $G_{0}$. The equation

$$
\int_{C} d 3 G^{-1}(1,3) G(3,2)=\int_{C} d 3 G(1,3) G^{-1}(3,2)=\delta(1,2)
$$

defines the inverse one-particle Green function. Here $\delta(1,2)$ denotes the product of a delta function in Minkowski space $\delta\left(x_{1}-x_{2}\right)$ times a Kronecker delta $\delta_{\alpha_{1} \alpha_{2}}$ for the spinor indices. Finally, the integral $\int_{C} d 3$ is a short-hand notation for $\sum_{\alpha_{3}} \int_{C} d t_{3} \int d^{3} r_{3}$. For spin- $\frac{1}{2}$ fields one finds

$$
G_{0}^{-1}(1,2)=\left(i \gamma_{\mu} \partial_{1}^{\mu}-m_{N}\right) \delta(1,2) .
$$

From the equations of motion for the field operators one obtains the Dyson equation for the one-particle Green function

$$
G(1,2)=G_{0}(1,2)+\int_{C} d 3 \int_{C} d 4 G_{0}(1,3) \Sigma(3,4) G(4,2)
$$


or equivalently

$$
G(1,2)=G_{0}(1,2)+\int_{C} d 3 \int_{C} d 4 G(1,3) \Sigma(3,4) G_{0}(4,2)
$$

where $\Sigma$ denotes the self-energy. In analogy to (20) one can define the correlation parts of the self-energy $\Sigma^{>}$and $\Sigma^{<}$such that

$$
\begin{aligned}
\Sigma(1,2)= & \Sigma^{\delta}(1,2) \delta\left(t_{1}-t_{2}\right) \\
& +\Theta_{C}\left(t_{1}-t_{2}\right) \Sigma^{>}(1,2)+\Theta_{C}\left(t_{2}-t_{1}\right) \Sigma^{<}(1,2) .
\end{aligned}
$$

The singular term $\Sigma^{\delta}(1,2) \delta\left(t_{1}-t_{2}\right)$ is due to interactions, which are local in time, i.e., where the two time arguments coincide on the contour $C$. In section 2.4 this part of the self-energy is identified with the mean-field contributions which are local both in space and time.

The retarded and advanced self-energies are given by

$$
\begin{aligned}
& \Sigma^{+}(1,2)=\Sigma^{\delta}(1,2) \delta\left(t_{1}-t_{2}\right)+\Theta_{C}\left(t_{1}-t_{2}\right)\left(\Sigma^{>}(1,2)-\Sigma^{<}(1,2)\right) \\
& \Sigma^{-}(1,2)=\Sigma^{\delta}(1,2) \delta\left(t_{1}-t_{2}\right)-\Theta_{C}\left(t_{2}-t_{1}\right)\left(\Sigma^{>}(1,2)-\Sigma^{<}(1,2)\right) .
\end{aligned}
$$

The self-energies satisfy relations analogous to those for the Green functions (21) and (22).

For the expression $\int_{C} d 3 G_{0}^{-1}(1,3) G(3,2)$, with the time arguments $t_{1}$ and $t_{2}$ on opposite branches of the contour $C$, the Dyson equation (26) yields the following equations of motion for the correlation functions $G^{>}$and $G^{<}$:

$$
\begin{aligned}
\left(i \gamma_{\mu} \partial_{1}^{\mu}-m_{N}\right) G^{<}(1,2) & =\int d^{3} r_{3} \Sigma^{\delta}\left(t_{1}, \boldsymbol{r}_{1} ; t_{1}, \boldsymbol{r}_{3}\right) G^{<}\left(t_{1}, \boldsymbol{r}_{3} ; 2\right) \\
& +\int_{t_{0}}^{t_{1}} d 3\left(\Sigma^{>}(1,3)-\Sigma^{<}(1,3)\right) G^{<}(3,2) \\
& -\int_{t_{0}}^{t_{2}} d 3 \Sigma^{<}(1,3)\left(G^{>}(3,2)-G^{<}(3,2)\right), \\
\left(i \gamma_{\mu} \partial_{1}^{\mu}-m_{N}\right) G^{>}(1,2) & =\int d^{3} r_{3} \Sigma^{\delta}\left(t_{1}, \boldsymbol{r}_{1} ; t_{1}, \boldsymbol{r}_{3}\right) G^{>}\left(t_{1}, \boldsymbol{r}_{3} ; 2\right) \\
& +\int_{t_{0}}^{t_{1}} d 3\left(\Sigma^{>}(1,3)-\Sigma^{<}(1,3)\right) G^{>}(3,2) \\
& -\int_{t_{0}}^{t_{2}} d 3 \Sigma^{>}(1,3)\left(G^{>}(3,2)-G^{<}(3,2)\right),
\end{aligned}
$$

where eqs. (24) and (25) have been taken into account. In these equations the time integrations are of the standard kind, i.e., only along the real axis. The equations (31) and (32) are known as the Kadanoff-Baym equations [16]. By 
using (18),(19) and the corresponding equations $(29),(30)$ for the self-energy one obtains the analogous equations of motion

$$
\int d 3 G_{0}^{-1}(1,3) G^{ \pm}(3,2)=\delta(1,2)+\int_{t_{0}}^{\infty} d 3 \Sigma^{ \pm}(1,3) G^{ \pm}(3,2)
$$

for the retarded and advanced Green functions.

\subsection{Gradient expansion and quasi-particle approximation}

As noted in the preceding section, one can distinguish local and non-local selfenergy contributions; the local ones are included in $\Sigma^{\delta}$ and the non-local ones in $\Sigma^{>}$ and $\Sigma^{<}$. The local part of the self-energy $\Sigma^{\delta}$ contains all mean-field contributions (tadpole diagrams) from the coupling of the nucleons to the scalar and vector mesons, and hence is of the form

$$
\Sigma^{\delta}\left(x_{1}, x_{2}\right)=I\left(\Sigma_{S}\left(x_{1}\right)+\gamma_{\mu} \Sigma_{V}^{\mu}\left(x_{1}\right)\right) \delta\left(\boldsymbol{r}_{1}-\boldsymbol{r}_{2}\right),
$$

where $I$ is the eight-by-eight unit matrix in the combined spinor and isospin space. The Lorentz-scalar part $\Sigma_{S}$ gives rise to time- and space-dependent effective masses of the nucleons, while the Lorentz-vector part $\Sigma_{V}^{\mu}$ adds to the momentum, thus yielding a kinetic momentum in analogy with classical electrodynamics. Higher order (non mean-field) diagrams contribute to the correlation parts $\Sigma^{>}$and $\Sigma^{<}$ in eqs. (31) and (32). In general, the corresponding diagrams yield both real and imaginary contributions to $\Sigma$.

We now substitute (34) in eq. (31) and transform to relative and CM coordinates and retain terms to linear order in a Taylor expansion around the CM coordinate $X=\frac{1}{2}\left(x_{1}+x_{2}\right)$. Finally, letting $t_{0} \rightarrow-\infty$ and taking the Fourier transform with respect to the relative coordinate, we obtain the equation of motion for the covariant Wigner transform of the correlation function $G^{<}$,

$$
\begin{aligned}
& \left\{\frac{i}{2} \gamma_{\mu} \partial_{X}^{\mu}+\gamma_{\mu}\left(p^{\mu}-\Sigma_{V}^{\mu}(X)\right)-m_{N}-\Sigma_{S}(X)\right. \\
& \left.+\frac{i}{2}\left(\gamma_{\mu} \partial_{X}^{\nu} \Sigma_{V}^{\mu}(X)+\partial_{X}^{\nu} \Sigma_{S}(X)\right) \partial_{\nu}^{p}\right\} i G^{<}(p, X)= \\
& \int d y \exp \{i p y\}\left[\int _ { - \infty } ^ { y _ { 0 } } d x ^ { \prime } \left\{\left(\Sigma^{>}-\Sigma^{<}\right)\left(y-x^{\prime}, X\right) i G^{<}\left(x^{\prime}, X\right)\right.\right. \\
& +\left(\frac{1}{2} x^{\prime} \partial_{X}\left(\Sigma^{>}-\Sigma^{<}\right)\left(y-x^{\prime}, X\right)\right) i G^{<}\left(x^{\prime}, X\right) \\
& \left.+\left(\Sigma^{>}-\Sigma^{<}\right)\left(y-x^{\prime}, X\right) \frac{1}{2}\left(x^{\prime}-y\right) \partial_{X} i G^{<}\left(x^{\prime}, X\right)\right\} \\
& -\int_{y_{0}}^{\infty} d x^{\prime}\left\{\Sigma^{<}\left(x^{\prime}, X\right)\left(i G^{>}-i G^{<}\right)\left(y-x^{\prime}, X\right)\right. \\
& +\left(\frac{1}{2}\left(y-x^{\prime}\right) \partial_{X} \Sigma^{<}\left(x^{\prime}, X\right)\right)\left(i G^{>}-i G^{<}\right)\left(y-x^{\prime}, X\right) \\
& \left.\left.+\Sigma^{<}\left(x^{\prime}, X\right) \frac{1}{2}\left(-x^{\prime}\right) \partial_{X}\left(i G^{>}-i G^{<}\right)\left(y-x^{\prime}, X\right)\right\}\right] \text {. }
\end{aligned}
$$


We note that the Green functions $G$ and the self-energies $\Sigma$ are matrices in spinor and isospin space. Thus, the ordering of the functions is important. The Taylor expansion in eq. (35) is known as the gradient expansion [16].

The equation of motion for $G^{>}$are obtained analogously. By taking the difference of eq. (35) and its adjoint, taking the trace with respect to spinor and isospin indices and performing the Fourier-transformation with respect to relative coordinates, we find the covariant kinetic equation

$$
\begin{aligned}
\operatorname{Tr} & \left\{i\left(\gamma_{\mu} \partial_{X}^{\mu}+\partial_{X}^{\nu} \Sigma^{\delta}(X) \partial_{\nu}^{p}\right) i G^{<}(p, X)\right\}= \\
& \operatorname{Tr}\left\{i\left(\Sigma^{>}(p, X) G^{<}(p, X)-\Sigma^{<}(p, X) G^{>}(p, X)\right)\right\} \\
& -\operatorname{Tr}\left\{\left[\operatorname{Re} \Sigma^{+}(p, X), G^{<}(p, X)\right]\right\} \\
& -\operatorname{Tr}\left\{\left[\Sigma^{<}(p, X), \operatorname{Re} G^{+}(p, X)\right]\right\} .
\end{aligned}
$$

Here

$$
[f, g]:=\left(\partial_{\nu}^{p} f\right) \partial_{X}^{\nu} g-\left(\partial_{X}^{\nu} f\right) \partial_{\nu}^{p} g
$$

denotes a Poisson bracket. Equation (36) is the relativistic generalization of the quantum-mechanical Boltzmann equation $[16,19]$ for fermions. The gradient expansion, which was used in its derivation, is valid if the Green function $G(p, X)$ and self-energy $\Sigma(p, X)$ vary slowly in space-time compared to the inverse of the typical momenta of the system $[15,29]$.

At this point we introduce the quasi-particle approximation, which involves an average of eq. (36) over an energy interval which is large compared to the inverse scattering rate (width of quasi-particle peak in the spectral function) and small compared to the characteristic single-particle energies of the system [19]. The quasi-particle approximation is valid for sufficiently dilute systems and for low temperature fermion systems. Whether it is applicable to such highly excited nuclear systems as those formed in intermediate energy heavy-ion collisions is in fact questionable. However, at present this is the only way which permits the study of realistic systems. Therefore, we explore solutions to the kinetic equations in this approximation.

The left-hand side of eq. (36) represents the covariant Vlasov-term [14], which describes the mean-field dynamics, while the effects of correlations are included in the terms on the right-hand side. The first term on the right-hand side of eq. (36) can be interpreted as the collision integral which changes the quasi-particle distribution in momentum space, while the second leads to a modification of the quasi-particle energies due to correlation effects.

In dilute systems with short-ranged interactions, the remaining terms on the right-hand side are small compared to the collision term [19]. Furthermore, the third term on the right-hand side of eq. (36) contains a gradient of the scattering rate, and hence is a higher-order contribution in the gradient expansion. Both of these terms are neglected here, in accordance with the quasiparticle approximation and the gradient expansion. 
The resulting kinetic equation then reads

$$
\begin{aligned}
& \operatorname{Tr}\left\{\left[\gamma_{\mu} p^{\mu}-\Sigma^{\delta}(X), i G^{<}(p, X)\right]\right\}= \\
& \quad \operatorname{Tr}\left\{\Sigma^{>}(p, X) G^{<}(p, X)-\Sigma^{<}(p, X) G^{>}(p, X)\right\} .
\end{aligned}
$$

With the help of Dirac matrices the correlation functions $G^{<}, G^{>}$and the selfenergy functions can be decomposed into scalar, vector, pseudo-scalar, pseudovector and tensor parts. Thus, e.g., the single-particle Green function $G^{<}$is of the form

$$
\begin{aligned}
-i G^{<}(p, X)= & I f_{S}(p, X)+\gamma_{\lambda} f_{V}^{\lambda}(p, X)+\gamma_{5} f_{P S}(p, X)+\gamma_{5} \gamma_{\lambda} f_{P V}^{\lambda}(p, X) \\
& +\frac{1}{2} \sigma_{\lambda \rho} f_{T}^{\lambda \rho}(p, X),
\end{aligned}
$$

where isospin indices are suppressed. In the following we shall restrict ourselves to spin and isospin-saturated and parity-invariant systems, where the pseudo-scalar, pseudo-vector and tensor parts vanish.

\subsection{Perturbation expansion for the self-energy}

In this section we derive perturbative expansions for the nucleon self-energy functions entering the kinetic equation (37). In analogy to the different types of oneparticle Green functions on the time contour, we distinguish between the correlation parts $\Sigma^{<}$and $\Sigma^{>}$as well as the retarded and advanced parts, $\Sigma^{+}$and $\Sigma^{-}$, of the self-energy. The calculations of the different self-energy terms are performed within the real-time approach to non-equilibrium systems [18-20]. Retaining only the lowest-order term in the gradient expansion amounts to a local density approximation. Thus, the Green functions and self-energies in eq. (37) are those of a homogeneous system, the state of which is that existing locally at the point $X$. Consequently, it is convenient to work in momentum space. For the lagrangian (1) we obtain the following Feynman rules for the terms of order $\mathrm{n}$.

- Draw all topologically distinct diagrams with two external propagator lines and $2 \mathrm{n}$ interaction vertices connected by internal propagator lines.

- Assign a direction to each line. Associate a directed four-momentum with each line and conserve the four-momentum at each vertex.

- For each directed line multiply by a factor $i$ times the propagator.

- Insert a causal or anti-causal propagator, respectively, if the starting and the ending points of the line are located on the same branch of the time contour.

- Insert the correlation parts of the propagators if the starting and end points of the line are on different branches of the time contour.

- For each vertex multiply by a spin and isospin matrix. For the mesonnucleon-nucleon vertices use $i g_{\sigma} I,-i g_{\omega} \gamma_{\mu} I$ and $g_{\pi} \gamma_{5} \vec{\tau}$ for a scalar, vector and pseudo-scalar meson, respectively. 
- Integrate over all independent four-momenta.

- Multiply with a factor -1 for each closed fermion loop.

The mean-field part of the self-energy $\Sigma^{\delta}$ is due to the tadpole diagrams with scalar and vector mesons [19]. The corresponding scalar and vector components of the self-energy defined in (34) are given by

$$
\begin{gathered}
\Sigma_{S}=i \frac{g_{\sigma}^{2}}{m_{\sigma}^{2}} \int \frac{d^{4} p}{(2 \pi)^{4}} \operatorname{Tr}\left\{G_{H}(p)\right\}, \\
\Sigma_{V}^{\mu}=-i \frac{g_{\omega}^{2}}{m_{\omega}^{2}} \int \frac{d^{4} p}{(2 \pi)^{4}} \operatorname{Tr}\left\{\gamma^{\mu} G_{H}(p)\right\},
\end{gathered}
$$

where $G_{H}$ is the Hartree propagator which satisfies the Dyson equation (26) with $\Sigma(1,2)=\Sigma^{\delta}(1,2) \delta\left(t_{1}-t_{2}\right)$. Thus we obtain the well-known self-consistency condition of the mean-field approximation [13].

In fig. 2 we show the lowest-order diagrams which contribute to the correlation parts $\Sigma^{>}$and $\Sigma^{<}$of the self-energy and thereby to the collision integrals of the kinetic equation (37). These diagrams correspond to the Born approximation for the $T$-matrix with the exchange of scalar, vector and pseudo-scalar mesons.

In the calculation of correlation diagrams, the nucleon lines are evaluated in the Hartree-approximation. Thus, it is convenient to include the effects of the mean fields $\Sigma_{S}$ and $\Sigma_{V}$ in the plane-wave decomposition of the nucleon fields :

$$
\begin{array}{r}
\Psi(x)=\int \frac{d^{3} p}{(2 \pi)^{3}} \sum_{s}\left(a(\boldsymbol{p}, s) u_{s}(\boldsymbol{p}) e^{-i\left(p-\Sigma_{V}\right) x}+\right. \\
\left.b^{\dagger}(\boldsymbol{p}, s) v_{s}(\boldsymbol{p}) e^{i\left(p+\Sigma_{V}\right) x}\right), \\
\bar{\Psi}(x)=\int \frac{d^{3} p}{(2 \pi)^{3}} \sum_{s}\left(a^{\dagger}(\boldsymbol{p}, s) \bar{u}_{s}(\boldsymbol{p}) e^{i\left(p-\Sigma_{V}\right) x}+\right. \\
\left.b(\boldsymbol{p}, s) \bar{v}_{s}(\boldsymbol{p}) e^{-i\left(p+\Sigma_{V}\right) x}\right) .
\end{array}
$$

Here $a(\boldsymbol{p}, s)$ and $b(\boldsymbol{p}, s)$ denote creation and annihilation operators for nucleons with momentum $\boldsymbol{p}$ and spin $s$. The quantities $u$ and $v$ denote the Dirac spinors [24]

$$
\begin{aligned}
& u_{s}(\boldsymbol{p})=\left(\frac{\omega_{\boldsymbol{p}}^{N}+m_{N}^{*}}{2 \omega_{\boldsymbol{p}}^{N}}\right)^{\frac{1}{2}}\left(\begin{array}{cc}
\chi_{s} & \\
\frac{\boldsymbol{\sigma} \boldsymbol{p}}{\omega_{\boldsymbol{p}}^{N}+m_{N}^{*}} & \chi_{s}
\end{array}\right) \\
& v_{s}(\boldsymbol{p})=\left(\frac{\omega_{\boldsymbol{p}}^{N}+m_{N}^{*}}{2 \omega_{\boldsymbol{p}}^{N}}\right)^{\frac{1}{2}}\left(\begin{array}{cc}
\frac{\boldsymbol{\sigma p}}{\omega_{\boldsymbol{p}}^{N}+m_{N}^{*}} & \chi_{s} \\
\chi_{s}
\end{array}\right) \text {, }
\end{aligned}
$$

where $s= \pm \frac{1}{2}, \chi_{+\frac{1}{2}}=\left(\begin{array}{l}1 \\ 0\end{array}\right), \chi_{-\frac{1}{2}}=\left(\begin{array}{l}0 \\ 1\end{array}\right), \sigma$ denotes the Pauli matrices and $m_{N}^{*}=$ $m_{N}-\Sigma_{S}$ the effective nucleon mass and $\omega_{\boldsymbol{p}}^{N}=\sqrt{\boldsymbol{p}^{2}+m_{N}^{* 2}}$. The Dirac spinors $u$ 
and $v$ satisfy the completeness relations

$$
\begin{aligned}
& \sum_{s} u_{s}(\boldsymbol{p}) \bar{u}_{s}(\boldsymbol{p})=\left(\frac{\gamma^{\mu} p_{\mu}+m_{N}^{*}}{2 \omega_{\boldsymbol{p}}^{N}}\right), \\
& \sum_{s} v_{s}(\boldsymbol{p}) \bar{v}_{s}(\boldsymbol{p})=\left(\frac{\gamma^{\mu} p_{\mu}-m_{N}^{*}}{2 \omega_{\boldsymbol{p}}^{N}}\right) .
\end{aligned}
$$

In the following, the negative-energy (antiparticle) solutions are dropped, because they are expected to play a minor role in the collision dynamics at incident energies around $E_{\text {lab }}=1 \mathrm{GeV}$. Using the plane-wave decomposition (41) for the nucleon field operators, we obtain the following expressions for the nucleon correlation Green functions in the mean-field approximation

$$
\begin{aligned}
& i G^{<}\left(p^{*}, t\right)=-2 \pi \sum_{s} u_{s}\left(\boldsymbol{p}^{*}\right) \bar{u}_{\boldsymbol{s}}\left(\boldsymbol{p}^{*}\right) \delta\left(p_{0}^{*}-\omega_{\boldsymbol{p}^{*}}^{N}\right) f_{S}\left(\boldsymbol{p}^{*}, t\right) \\
& i G^{>}\left(\boldsymbol{p}^{*}, t\right)=+2 \pi \sum_{\boldsymbol{s}} u_{\boldsymbol{s}}\left(\boldsymbol{p}^{*}\right) \bar{u}_{\boldsymbol{s}}\left(\boldsymbol{p}^{*}\right) \delta\left(p_{0}^{*}-\omega_{\boldsymbol{p}^{*}}^{N}\right)\left(1-f_{S}\left(\boldsymbol{p}^{*}, t\right)\right),
\end{aligned}
$$

where $p^{*}=p-\Sigma_{V}$ denotes the kinetic momentum and $f_{S}$ the distribution function of the (mean-field) quasi-particles in the seven-dimensional phase space. By inserting the expressions for $G^{>}$and $G^{<}$into the self-energy diagrams of fig. 2, we find (cf. appendix) that the term $\operatorname{Tr}\left\{\Sigma^{<} G^{>}\right\}$in eq. (37) corresponds to the gain term, while $\operatorname{Tr}\left\{\Sigma^{>} G^{<}\right\}$corresponds to the loss term. Furthermore, we then recover the well-known Nordheim or Uehling-Uhlenbeck form [2,3] for the collision integral, modified by the medium dependence of the nucleon-nucleon scattering amplitude.

For spatially homogeneous systems the kinetic equation (37) for the phasespace density of the nucleons reduces to

$$
\partial_{t} f_{S}\left(\boldsymbol{p}^{*}, t\right)=\frac{1}{4} \operatorname{Tr} \int_{0}^{\infty} \frac{d p_{0}^{*}}{2 \pi}\left\{\Sigma^{<}\left(p^{*}, t\right) G^{>}\left(p^{*}, t\right)-\Sigma^{>}\left(p^{*}, t\right) G^{<}\left(p^{*}, t\right)\right\}
$$

with the correlation Green functions given by eqs. (43) and (44).

\subsection{Covariant kinetic equations for deltas}

In the preceding sections we have restricted our considerations to nucleons. Rather than repeating all detailed steps in the derivation of kinetic equations for deltas, we shall emphasize only the changes due to the differences in spin and isospin.

The inelastic nucleon-nucleon cross section at energies around $E_{\text {lab }}=1 \mathrm{GeV}$ is mainly due to the excitation of delta resonances which subsequently decay into nucleons and pions. Thus, for a realistic description of intermediate-energy heavyion collisions it is essential to include the effects of the delta resonance in the kinetic equations. Here we introduce the delta as an explicit degree of freedom. As far as we know, this approach has been used by all groups working in this field.

In the lagrangian (1) we have introduced the delta as an elementary baryonic degree of freedom with $J^{\pi}=\frac{3}{2}^{+}$and $T=\frac{3}{2}$. The Rarita-Schwinger-formalism [23] allows one to describe particles with higher spins in terms of the conventional 
four-component Dirac-theory for spin- $\frac{1}{2}$ particles. The spin- $\frac{3}{2}$ vector-spinor field $\Psi_{\Delta}^{\mu}$ is constructed from a spin-1 vector field and a spin- $\frac{1}{2}$ spinor field. Following the phase convention of ref. [25], the four spin components are described by the vector-spinors

$$
\begin{array}{ll}
w_{+\frac{3}{2}}^{\mu}=e_{+1}^{\mu} u_{+\frac{1}{2}}, & w_{+\frac{1}{2}}^{\mu}=\frac{1}{\sqrt{3}} e_{+1}^{\mu} u_{-\frac{1}{2}}+\left(\frac{2}{3}\right)^{\frac{1}{2}} e_{0}^{\mu} u_{+\frac{1}{2}}, \\
w_{-\frac{3}{2}}^{\mu}=e_{-1}^{\mu} u_{-\frac{1}{2}}, & w_{-\frac{1}{2}}^{\mu}=\frac{1}{\sqrt{3}} e_{-1}^{\mu} u_{+\frac{1}{2}}+\left(\frac{2}{3}\right)^{\frac{1}{2}} e_{0}^{\mu} u_{-\frac{1}{2}}
\end{array}
$$

with the spin-1 polarization vectors ( $z$-axis parallel to momentum $\boldsymbol{p}$ )

$$
\begin{aligned}
e_{+1}^{\mu} & =-\left(0, \frac{1}{\sqrt{2}}, \frac{i}{\sqrt{2}}, 0\right), \\
e_{-1}^{\mu} & =\left(0, \frac{1}{\sqrt{2}}, \frac{-i}{\sqrt{2}}, 0\right), \\
e_{0}^{\mu} & =\left(\frac{|\boldsymbol{p}|}{m_{\Delta}}, 0,0, \frac{\omega_{\boldsymbol{p}}^{\Delta}}{m_{\Delta}}\right)
\end{aligned}
$$

and the spinors

$$
u_{s}(\boldsymbol{p})=\left(\frac{\omega_{\vec{p}}^{\Delta}+m_{\Delta}}{2 \omega_{\boldsymbol{p}}^{\Delta}}\right)^{\frac{1}{2}}\left(\begin{array}{cc}
\chi_{s} \\
\frac{\boldsymbol{\sigma} \boldsymbol{p}}{\omega_{\hat{\boldsymbol{p}}}^{\Delta}+m_{\Delta}} & \chi_{s}
\end{array}\right) .
$$

The vector spinors satisfy the norrialization conditions

$$
\begin{aligned}
& \left(\bar{w}_{s}\right)_{\mu}\left(w_{s^{\prime}}\right)^{\mu}=-\delta_{s s^{\prime}} \frac{m_{\Delta}}{\omega_{\vec{p}}^{\Delta}}, \\
& \left(w_{s}^{\dagger}\right)_{\mu}\left(w_{s^{\prime}}\right)^{\mu}=-\delta_{s s^{\prime}} .
\end{aligned}
$$

and the completeness relation [26]

$$
\begin{aligned}
\sum_{\boldsymbol{s}} w_{s}^{\mu}(\boldsymbol{p}) \bar{w}_{s}^{\nu}(\boldsymbol{p})= & \frac{\gamma_{\mu} p^{\mu}+m_{\Delta}}{2 \omega_{\vec{p}}^{\Delta}}\left\{-g^{\mu \nu}+\frac{1}{3} \gamma^{\mu} \gamma^{\nu}+\frac{2}{3 p^{2}} p^{\mu} p^{\nu}\right\} \\
& +\frac{\gamma_{\mu} p^{\mu} m_{\Delta}+p^{2}}{6 \omega_{\vec{p}}^{\Delta} p^{2}}\left(\gamma^{\mu} p^{\nu}-\gamma^{\nu} p^{\mu}\right) .
\end{aligned}
$$

The coupling term of nucleons, deltas and pions in the lagrangian (1) also takes into account the different isospin of nucleons and deltas by introducing the isospin transition matrices [22] $\vec{T}=\left(T_{1}, T_{2}, T_{3}\right)$ which satisfy $\left(T_{j}^{\dagger} T_{i}\right)=\left(\delta_{j i}-\frac{1}{3} \sigma_{j} \sigma_{i}\right)$.

The one-particle correlation Green functions $G_{\mu \nu}^{>}$and $G_{\mu \nu}^{<}$for the deltas are defined like the correlation functions $G^{>}$and $G^{<}$for the nucleons. Using the quasiparticle approximation and the plane-wave decomposition in the mean-field basis, we obtain for a translationally invariant system

$$
\begin{aligned}
-i G_{\mu \nu}^{<}\left(p^{*}, t\right) & =-2 \pi \sum_{s} w_{s \mu}\left(\boldsymbol{p}^{*}\right) \bar{w}_{s \nu}\left(\boldsymbol{p}^{*}\right) \delta\left(p_{0}^{*}-\omega_{\boldsymbol{p}^{*}}^{\Delta}\right) f_{S}^{\Delta}\left(\boldsymbol{p}^{*}, t\right) \\
-i G_{\mu \nu}^{>}\left(p^{*}, t\right) & =+2 \pi \sum_{s} w_{s \mu}\left(\boldsymbol{p}^{*}\right) \bar{w}_{s \nu}\left(\boldsymbol{p}^{*}\right) \delta\left(p_{0}^{*}-\omega_{\boldsymbol{p}^{*}}^{\Delta}\right)\left(1-f_{S}^{\Delta}\left(\boldsymbol{p}^{*}, t\right)\right) .
\end{aligned}
$$


Here, $m_{\Delta}^{*}=m_{\Delta}+\Sigma_{S}$ and $p_{\mu}^{*}=p_{\mu}-\Sigma_{V \mu}$ denote the effective mass and the kinetic momentum of the deltas, respectively, while $f_{S}^{\Delta}\left(\boldsymbol{p}^{*}, t\right)$ is the scalar phase-space distribution function on the energy shell $\omega_{p^{*}}^{\Delta}=\sqrt{\boldsymbol{p}^{* 2}+m_{\Delta}^{* 2}}$. From the KadanoffBaym-equations for the deltas we finally obtain (using the gradient expansion and the quasi-particle approximation) the kinetic equation in a homogeneous system

$$
\partial_{t} f_{S}^{\Delta}\left(\boldsymbol{p}^{*}, t\right)=\frac{1}{16} \operatorname{Tr} \int_{0}^{\infty} \frac{d p_{0}^{*}}{2 \pi}\left\{\Sigma_{\mu \rho}^{<}\left(p^{*}, t\right) G_{\rho \nu}^{>}\left(p^{*}, t\right)-\Sigma_{\mu \rho}^{>}\left(p^{*}, t\right) G_{\rho \nu}^{<}\left(p^{*}, t\right)\right\}
$$

where the correlation Green functions are given by eqs. (48) and (49). Equations (45) and (50) will be used in the numerical application described in section 3.

Diagrams, which contain two delta lines, are neglected in the calculation of the self-energy, because of the small probability of delta-delta collisions at intermediate energies. Taking the pion-nucleon-delta coupling into account, we obtain additional rules for the evaluation of the self-energy:

- For each directed line of a delta resonance multiply by a factor $-i$ times the propagator.

- For each pion-nucleon-delta with an ingoing delta vertex multiply by the spin-isospin matrix $i g_{\pi N \Delta} K_{\mu} \vec{T}$ with $K_{\mu}$ denoting the four-momentum carried by an incoming pion and $g_{\pi N \Delta}=f_{\pi N \Delta} / m_{\pi}$.

- At a vertex with an outgoirg delta replace $\vec{T}$ by $\vec{T}^{\dagger}$.

The inclusion of deltas implies not only an additional kinetic equation (50), but also leads to modifications of the nucleon collision integral in equation (45). The Born diagrams for the elastic and inelastic collisions are shown in figs. 9-11 and the corresponding analytic expressions for the collision integrals are given in the appendix.

\section{Equilibration in dense nuclear matter}

We now explore the global characteristics of the relaxation process in nucleusnucleus collisions by solving the coupled kinetic equations (45) and (50) in a spatially homogeneous system with a non-equilibrium momentum distribution. The initial conditions are chosen such that the momentum distribution resembles that of a central nucleus-nucleus collision, i.e., as two counter-streaming nuclear-matter currents. This simple model is well suited for addressing the problem of equilibration in heavy-ion collisions, and furthermore, it can be employed as a testing ground for various approximations which are necessary in more realistic simulations of nucleus-nucleus collisions [11]. Our main interest here is to study typical medium effects in the relaxation process. 


\subsection{The nucleon-nucleon cross section}

The free parameters in the lagrangian (1) are the hadron masses and the baryonmeson coupling constants. For the masses we choose the conventional values $m_{N}=939 \mathrm{MeV}, m_{\Delta}=1232 \mathrm{MeV}, m_{\sigma}=550 \mathrm{MeV}, m_{\omega}=784 \mathrm{MeV}$ and $m_{\pi}=139 \mathrm{MeV}$. We allow for two sets of coupling constants; one to describe the mean-field properties of nuclear matter, and another one to fit the nucleon-nucleon scat iering cross sections in the Born approximation. Since a given higher-order contribution to the mean field has a different symmetry factor than the corresponding contribution to the scattering amplitude, it is not surprising that, within an effective theory, the values of the coupling constants, which are needed for describing the mean-field, differ from the ones fitted to the scattering cross section.

For the mean-field part we employ coupling constants adjusted to the groundstate properties of nuclear matter (cf. subsect. 3.2), while the coupling constants, employed in the elastic part of the collision term, are determined by fitting the elastic differential nucleon-nucleon cross section at an incident energy of $E_{\text {lab }}=$ $1 \mathrm{GeV}$. Finally, the $\pi N \Delta$-coupling constant is determined by the free decay width of the delta resonance [21].

The finite size of the baryons is taken into account by introducing form factors $F\left(q^{2}\right)$, where $q$ is the momentum transfer at the meson-baryon vertices. The formfactors are also crucial for obtaining reasonable agreement with the scattering data. In the elastic scattering channels we use $F=\Lambda^{2} /\left(\Lambda^{2}-q^{2}\right)$, and in the inelastic ones $F=\left[\Lambda^{2} /\left(\Lambda^{2}-q^{2}\right)\right]^{2}$, cf. ref. [25]. The numerical values for the coupling constants and the cut-off parameter $\Lambda$ are given in table 2.

In fig. 3 we illustrate how well the calculated elastic differential nucleon-nucleon cross section at $1 \mathrm{GeV}$ incident energy reproduces a fit by Cugnon [1] to experimental data. We note that with decreasing energies the differential cross section becomes more isotropic, whereas it becomes more peaked in forward direction with increasing energy, which is in qualitative agreement with experiment [27]. Also shown in fig. 3 is the cross section which is obtained by replacing the bare nucleon mass by an effective value $m_{N}^{*}=0.85 m_{N}$. With decreasing $m_{N}^{*}$ the cross section is enhanced for large scattering angles, an effect which shortens the equilibration time as discussed in the following subsection. In fig. 4 we compare the total elastic nucleon-nucleon cross section as a function of $E_{\text {lab }}$ for $m_{N}^{*}=m_{N}$ and $m_{N}^{*}=0.85 m_{N}$, respectively, with experimental data. For $m_{N}^{*}=m_{N}$ the energy dependence of the observed elastic nucleon-nucleon cross section is well reproduced. Increasing discrepancies are observed above $1 \mathrm{GeV}$, the difference reaching $10 \mathrm{mb}$ around $2.5 \mathrm{GeV}$. As expected from fig. 3 the cross section for $m_{N}^{*}=0.85 m_{N}$ is considerably higher.

The cross sections for inelastic scattering are also strongly enhanced by medium effects. A detailed discussion of the in-medium cross section in these channels is given in ref. [11].

An accurate study of the effective cross section in cold nuclear matter has been performed by ter Haar and Malfliet [28] within the Dirac-Brueckner approach using one boson-exchange potentials. An interesting result is that the effective NN cross 
section is considerably reduced in the energy range between $50 \mathrm{MeV}$ and $400 \mathrm{MeV}$ even if Pauli blocking in the final states is neglected. This effect is most likely due to Pauli blocking in the intermediate states of the ladders summed in the DiracBrueckner approach. At $1 \mathrm{GeV}$ this mechanism is still active and, compared to our results, leads to a weaker increase of the effective cross section with increasing $m_{N}^{*}$. It is unclear to us to what extent other differences in the treatment of the NN interaction, implying quite different off-shell behaviours, could also be responsible for the different $m_{N}^{*}$ dependence of the effective cross section. Within our kinetic model, treating mesons and baryons on the same footing, it is prohibitively difficult to go beyond the Born approximation. However around $1 \mathrm{GeV} / \mathrm{u}$, we expect that qualitative features of medium effects on the equilibration process in relativistic heavy-ion collisions can be revealed in our approach.

\subsection{Counter-streaming nuclear-matter currents}

We apply the kinetic equations (45) and (50) to the model of two counter-streaming nuclear-matter currents with the aim to gain insight into global features of the relaxation process and, in particular, to study effects which result from the mean mesonic fields and from medium corrections in the collision integrals.

The calculations are performed for initial conditions which correspond to a heavy-ion collision at a bombarding energy $E_{\text {lab }}=1 \mathrm{GeV} / \mathrm{u}$. These initial conditions for the two counter-streaming currents are related to a symmetric nucleusnucleus collision in the following way. At a bombarding energy of $E_{\text {lab }}=1 \mathrm{GeV} / \mathrm{u}$ the momentum distribution of the two nuclei, before they collide, can be represented by two Fermi spheres which are boosted with velocities $v_{z}= \pm 0.6 \mathrm{c}$ in the $\mathrm{CM}$ frame. However, more relevant for the relaxation process, and thus for our model, is the situation where the nuclei overlap. Then, the local density is more than doubled, which in turn modifies the mean fields. Now, in order to retain the relation between the initial conditions and the bombarding energy, the Fermi spheres of the initial state must be shifted. The shift is determined by requiring that energy, momentum and baryon number is conserved [29]. Thus, the initial conditions are determined by a collisionless shock front between the non-overlapping and overlapping regimes. Depending on the strength of the mean fields, the initial conditions differ. Only after the mean fields are fixed, the relaxation process is allowed to begin. We cannot justify this procedure; however, there seems no alternative to exist, which consistently takes care of the conservation laws.

We have studied the time evolution of the equilibration process using three different sets of mean-field coupling constants. In model (i) we use the original Walecka model [13] which yields a large compression modulus $K_{0}=540 \mathrm{MeV}$ and a small effective mass $m_{N 0}^{*}=0.56 m_{N}$ for nuclear matter at equilibrium density $\rho_{0}$. In model (ii) a more realistic mean-field model is used which includes selfinteractions

$$
\delta \mathcal{L}=\frac{1}{3} b \Phi^{3}+\frac{1}{4} c \Phi^{4}
$$


of the $\sigma$-field and yields the more realistic values $K_{0}=210 \mathrm{MeV}$ and $m_{N 0}^{*}=$ $0.85 m_{N}$ for the compression modulus and the effective mass [30]. The two sets of coupling constants are given in table 3. Finally, in order to have a reference point, we have also done calculations neglecting the mean fields in all expressions (version (iii)).

In fig. 5 we show contour plots of the momentum distribution obtained with the Walecka mean-field model (version(i)) as a function of time. The nucleon distribution function is shown in the upper part, while that of the deltas is shown in the lower part. In this model, the system equilibrates very fast; after $2 \mathrm{fm} / \mathrm{c}$ the system is already close to thermal equilibrium. In figs. 6 and 7 the corresponding plots are shown for versions (ii) and (iii), respectively. Both of these versions yield equilibration times $(4 \mathrm{fm} / \mathrm{c}$ and $8 \mathrm{fm} / \mathrm{c}$ ) which are considerably larger than those of version (i).

There are two important effects involving the mean fields, which leads to the vastly different equilibration times for the different mean-field assumptions.

(a) The modification of the mean fields, once the colliding systems are superimposed, leads to a shift of the Fermi spheres in the initial state as explained above. Due to this shift, which in model (i) is fairly large, the initial momentum distributions are located closer to each other than when the mean fields are neglected, as in model (iii). Consequently, the average relative momenta of scattering particles in (ii) and, in particular, in (i) are smaller than in (iii). Thus, the almost isotropic cross section at low relative momenta allows particles to scatter to large angles more easily. The equilibration is faster, because the Pauli blocking of particles scattered to large angles is reduced, compared to scattering with a forward-peaked cross section.

(b) Furthermore, as was demonstrated above, the nucleon-nucleon cross section is strongly dependent on the baryon effective masses in medium. As compared to the free nucleon-nucleon cross section, the in-medium cross section favours large scattering angles. Thus, again scattering to large angles is possible, implying faster equilibration.

In addition to the thermal equilibration we have studied the relaxation towards chemical equilibrium. In fig. 8 the densities of nucleons and deltas for the three models (i) to (iii) are shown as function of time. Chemical equilibrium is achieved after approximately $3 \mathrm{fm} / \mathrm{c}$ in all models. Thus, in contrast to thermalization, chemical equilibration is not depending on medium effects. The final relative delta abundances $\rho_{\Delta} / \rho_{B}$ for (i) to (iii) are $0.35,0.26$ and 0.27 , respectively.

\section{Discussion and conclusion}

The treatment of two counter-streaming nuclear-matter currents has shown that the equilibration process in a heavy-ion collision at intermediate energies is strongly affected by medium effects which result from the large scalar and vector fields. Of course, in nucleus-nucleus collisions no homogeneous system exists, and thus, we should not compare these results quantitatively with experimental data. For ex- 
ample, as compared to ref. [32] the abundance of deltas at equilibrium turns out to be rather insensitive to the mean-field model (cf. the discussion at the end of the preceding section). The reason is the absence of dynamical compression in the homogeneous system. In ref. [32] the inclusion of compression within a onedimensional shock approximation yields large differences in the delta abundances for different mean-field models.

For a realistic treatment of heavy-ion collisions it is necessary to solve the relativistic Landau-Vlasov equations for the seven-dimensional (time plus six components of position and momentum) phase-space distribution function. However, as illustrated in the appendix, the relativistic collision terms become quite involved already in the Born approximation, and hence a numerical solution for realistic heavy-ion collisions cannot be obtained with available computers. In practicable calculations, therefore, simple parametrizations of the collision terms - frequently even without medium corrections - are applied.

An alternative way to reduce the numerical effort is to treat only moments (in momentum space) of the distribution functions, thereby decreasing the number of dynamical degrees of freedom. Such an approach is employed e.g. in the derivation of two- or three-fluid models which still contain the essential effects of relativistic mean fields and medium corrections of the collision terms [11]. The relativistic Landau-Vlasov equation, as presented here, is a good starting point for such developments.

However, usually one cannot rigorously justify all approximations involved in the derivation of such models. Therefore, detailed tests by comparison with exact solutions are necessary. In this respect the model of two counter-streaming nuclear-matter currents - tractable within the relativistic Landau-Vlasov equations - represents an ideal testing ground for such new developments. The results, reported here for different models of QHD, may be useful in this connection.

We thank C.M. Ko, R. Malfliet and St. Mrówczyński for fruitful discussions. Two of the authors (M.C. and M.S.) acknowledge financial support by the Friedrich Naumann Foundation.

\section{Appendix: Collision terms}

In fig. 2 we have introduced the self-energy diagrams which correspond to the Born approximation for the scattering amplitude and contribute to the elastic part of the collision integral. From these Born diagrams we obtain the following analytic expression for the gain term in eq. (45)

$$
\begin{aligned}
\operatorname{Tr}\left\{\Sigma^{<} G^{>}\right\}= & \int \frac{d^{3} p_{2}^{*}}{(2 \pi)^{3}} \int \frac{d^{3} p_{3}^{*}}{(2 \pi)^{3}} \int \frac{d^{3} p_{4}^{*}}{(2 \pi)^{3}}(2 \pi)^{4} \delta^{4}\left(p^{*}+p_{2}^{*}-p_{3}^{*}-p_{4}^{*}\right) \\
& \frac{1}{\omega \omega_{2} \omega_{3} \omega_{4}} f_{S}\left(\boldsymbol{p}_{3}^{*}\right) f_{S}\left(\boldsymbol{p}_{4}^{*}\right)\left(1-f_{S}\left(\boldsymbol{p}_{2}^{*}\right)\right)\left(1-f_{S}\left(\boldsymbol{p}^{*}\right)\right)
\end{aligned}
$$




$$
\begin{aligned}
& \left\{g _ { \sigma } ^ { 4 } \frac { 4 } { ( ( p ^ { * } - p _ { 3 } ^ { * } ) ^ { 2 } - m _ { S } ^ { 2 } ) ^ { 2 } } \left[m_{N}^{* 4}+\right.\right. \\
& \left.m_{N}^{* 2}\left(p^{*} p_{3}^{*}\right)+m_{N}^{* 2}\left(p_{2}^{*} p_{4}^{*}\right)+\left(p^{*} p_{3}^{*}\right)\left(p_{2}^{*} p_{4}^{*}\right)\right] \\
& -\frac{1}{2} g_{\sigma}^{4} \frac{1}{\left(\left(p^{*}-p_{3}^{*}\right)^{2}-m_{S}^{2}\right)\left(\left(p^{*}-p_{4}^{*}\right)^{2}-m_{S}^{2}\right)}\left[m_{N}^{* 4}+\right. \\
& \left(p^{*} p_{4}^{*}\right)\left(p_{2}^{*} p_{3}^{*}\right)-\left(p^{*} p_{2}^{*}\right)\left(p_{3}^{*} p_{4}^{*}\right)+\left(p^{*} p_{3}^{*}\right)\left(p_{2}^{*} p_{4}^{*}\right)+ \\
& m_{N}^{* 2}\left\{\left(p_{2}^{*} p_{3}^{*}\right)+\left(p^{*} p_{4}^{*}\right)+\left(p^{*} p_{3}^{*}\right)+\left(p_{3}^{*} p_{4}^{*}\right)+\right. \\
& \left.\left.\left(p^{*} p_{2}^{*}\right)+\left(p_{2}^{*} p_{4}^{*}\right)\right\}\right] \\
& +g_{\omega}^{4} \frac{8}{\left(\left(p^{*}-p_{3}^{*}\right)^{2}-m_{V}^{2}\right)^{2}}\left[2 m_{N}^{* 4}-m_{N}^{* 2}\left(p^{*} p_{3}^{*}\right)-\right. \\
& \left.m_{N}^{* 2}\left(p_{2}^{*} p_{4}^{*}\right)+\left(p^{*} p_{2}^{*}\right)\left(p_{3}^{*} p_{4}^{*}\right)+\left(p^{*} p_{4}^{*}\right)\left(p_{2}^{*} p_{3}^{*}\right)\right] \\
& -2 g_{\omega}^{4} \frac{1}{\left(\left(p^{*}-p_{3}^{*}\right)^{2}-m_{V}^{2}\right)\left(\left(p^{*}-p_{4}^{*}\right)^{2}-m_{V}^{2}\right)} \\
& {\left[-2 m_{N}^{* 4}-2\left(p^{*} p_{2}^{*}\right)\left(p_{3}^{*} p_{4}^{*}\right)+m_{N}^{* 2}\left\{\left(p_{2}^{*} p_{3}^{*}\right)+\right.\right.} \\
& \left.\left.\left(p^{*} p_{4}^{*}\right)+\left(p^{*} p_{3}^{*}\right)+\left(p_{3}^{*} p_{4}^{*}\right)+\left(p^{*} p_{2}^{*}\right)+\left(p_{2}^{*} p_{4}^{*}\right)\right\}\right] \\
& -8 g_{\sigma}^{2} g_{\omega}^{2} \frac{1}{\left(\left(p^{*}-p_{3}^{*}\right)^{2}-m_{S}^{2}\right)\left(\left(p^{*}-p_{3}^{*}\right)^{2}-m_{V}^{2}\right)} \\
& {\left[m_{N}^{* 2}\left\{\left(p^{*} p_{2}^{*}\right)+\left(p^{*} p_{4}^{*}\right)+\left(p_{2}^{*} p_{3}^{*}\right)+\left(p_{3}^{*} p_{4}^{*}\right)\right\}\right]} \\
& +2 g_{\sigma}^{2} g_{\omega}^{2} \frac{1}{\left(\left(p^{*}-p_{3}^{*}\right)^{2}-m_{S}^{2}\right)\left(\left(p^{*}-p_{4}^{*}\right)^{2}-m_{V}^{2}\right)} \\
& {\left[2 m_{N}^{* 4}+2\left(p^{*} p_{3}^{*}\right)\left(p_{2}^{*} p_{4}^{*}\right)+m_{N}^{* 2}\left\{2\left(p_{2}^{*} p_{4}^{*}\right)+\right.\right.} \\
& \left.\left.2\left(p^{*} p_{3}^{*}\right)-\left(p^{*} p_{4}^{*}\right)-\left(p_{3}^{*} p_{4}^{*}\right)-\left(p^{*} p_{2}^{*}\right)-\left(p_{2}^{*} p_{3}^{*}\right)\right\}\right] \\
& +g_{\pi}^{4} \frac{12}{\left(\left(p^{*}-p_{3}^{*}\right)^{2}-m_{\pi}^{2}\right)^{2}}\left[m_{N}^{* 4}-\right. \\
& \left.m_{N}^{* 2}\left(p^{*} p_{3}^{*}\right)-m_{N}^{* 2}\left(p_{2}^{*} p_{4}^{*}\right)+\left(p^{*} p_{3}^{*}\right)\left(p_{2}^{*} p_{4}^{*}\right)\right] \\
& +\frac{3}{2} g_{\pi}^{4} \frac{1}{\left(\left(p^{*}-p_{3}^{*}\right)^{2}-m_{\pi}^{2}\right)\left(\left(p^{*}-p_{4}^{*}\right)^{2}-m_{\pi}^{2}\right)}\left[m_{N}^{* 4}+\right. \\
& \left(p^{*} p_{4}^{*}\right)\left(p_{2}^{*} p_{3}^{*}\right)-\left(p^{*} p_{2}^{*}\right)\left(p_{3}^{*} p_{4}^{*}\right)+\left(p^{*} p_{3}^{*}\right)\left(p_{2}^{*} p_{4}^{*}\right)- \\
& m_{N}^{* 2}\left\{\left(p_{2}^{*} p_{3}^{*}\right)+\left(p^{*} p_{3}^{*}\right)+\left(p^{*} p_{4}^{*}\right)+\left(p_{2}^{*} p_{4}^{*}\right)-\right. \\
& \left.\left.\left(p^{*} p_{2}^{*}\right)-\left(p_{3}^{*} p_{4}^{*}\right)\right\}\right] \\
& +3 g_{\pi}^{2} g_{\sigma}^{2} \frac{1}{\left(\left(p^{*}-p_{3}^{*}\right)^{2}-m_{\pi}^{2}\right)\left(\left(p^{*}-p_{4}^{*}\right)^{2}-m_{S}^{2}\right)}\left[m_{N}^{* 4}+\right. \\
& \left(p^{*} p_{4}^{*}\right)\left(p_{2}^{*} p_{3}^{*}\right)+\left(p^{*} p_{3}^{*}\right)\left(p_{2}^{*} p_{4}^{*}\right)-\left(p^{*} p_{2}^{*}\right)\left(p_{3}^{*} p_{4}^{*}\right)+ \\
& m_{N}^{* 2}\left\{\left(p_{2}^{*} p_{3}^{*}\right)+\left(p^{*} p_{4}^{*}\right)-\left(p^{*} p_{3}^{*}\right)-\left(p_{3}^{*} p_{4}^{*}\right)-\right. \\
& \left.\left.\left(p^{*} p_{2}^{*}\right)-\left(p_{2}^{*} p_{4}^{*}\right)\right\}\right] \\
& -6 g_{\pi}^{2} g_{\omega}^{2} \frac{1}{\left(\left(p^{*}-p_{3}^{*}\right)^{2}-m_{\pi}^{2}\right)\left(\left(p^{*}-p_{4}^{*}\right)^{2}-m_{V}^{2}\right)}\left[-2 m_{N}^{* 4}+\right. \\
& m_{N}^{* 2}\left\{\left(p_{2}^{*} p_{3}^{*}\right)+\left(p^{*} p_{4}^{*}\right)-\left(p^{*} p_{2}^{*}\right)-\left(p_{3}^{*} p_{4}^{*}\right)+\right. \\
& \left.\left.\left.2\left(p^{*} p_{3}^{*}\right)+2\left(p_{2}^{*} p_{4}^{*}\right)-2\left(p^{*} p_{3}^{*}\right)\left(p_{2}^{*} p_{4}^{*}\right)\right\}\right]\right\} \text {, }
\end{aligned}
$$


where the abbreviation $(p q)$ denotes the scalar product of the four-vectors $p$ and $q$.

In figs. 9 and 10, respectively, the direct and exchange diagrams of the inelastic processes are given, which contribute to $-i \Sigma^{<}$in the kinetic equation for the nucleons. In fig. 11 the diagrams are shown, which determine the collision integral of the kinetic equation for the deltas. We illustrate the corresponding analytical expressions by the following two examples. The diagram (b) in fig. 9 contributes the expression

$$
\begin{aligned}
\operatorname{Tr}\left\{\Sigma_{(9 b)}^{<} G_{N}^{>}\right\}= & \int \frac{d^{3} p_{2}^{*}}{(2 \pi)^{3}} \int \frac{d^{3} p_{3}^{*}}{(2 \pi)^{3}} \int \frac{d^{3} p_{4}^{*}}{(2 \pi)^{3}}(2 \pi)^{4} \delta^{4}\left(p^{*}+p_{2}^{*}-p_{3}^{*}-p_{4}^{*}\right) \\
& \frac{1}{\omega \omega_{2} \omega_{3} \omega_{4}^{\Delta}} f_{S}\left(\boldsymbol{p}_{3}^{*}\right) f_{S}^{\Delta}\left(\boldsymbol{p}_{4}^{*}\right)\left(1-f_{S}\left(\boldsymbol{p}_{2}^{*}\right)\right)\left(1-f_{S}\left(\boldsymbol{p}^{*}\right)\right) \\
& \left\{\left(g_{\pi} \frac{f_{\pi N \Delta}}{m_{\pi}}\right)^{2} 8 \frac{1}{\left(\left(p^{*}-p_{3}^{*}\right)^{2}-m_{\pi}^{2}\right)^{2}}\right. \\
& {\left[\left(\left(p_{2}^{*} p_{4}^{*}\right)+m_{N}^{*} m_{\Delta}^{*}\right)\left(\left(p^{*} p_{3}^{*}\right)-m_{N}^{* 2}\right)\right.} \\
& \left.\left.\left(\frac{2}{3 m_{\Delta}^{* 2}}\left(p_{4}^{*} K\right)^{2}-\frac{2}{3} K^{2}\right)\right]\right\}
\end{aligned}
$$

to the nucleon gain term and corresponds to the reaction $N \Delta \rightarrow N N$. The diagram (b) of fig. 11 contributes

$$
\begin{aligned}
\operatorname{Tr}\left\{\Sigma_{(11 b)}^{<} G_{\Delta}^{>}\right\}= & \int \frac{d^{3} p_{2}^{*}}{(2 \pi)^{3}} \int \frac{d^{3} p_{3}^{*}}{(2 \pi)^{3}} \int \frac{d^{3} p_{4}^{*}}{(2 \pi)^{3}}(2 \pi)^{4} \delta^{4}\left(p^{*}+p_{2}^{*}-p_{3}^{*}-p_{4}^{*}\right) \\
& \frac{1}{\omega^{\Delta} \omega_{2}^{\Delta} \omega_{3} \omega_{4}} f_{S}\left(\boldsymbol{p}_{3}^{*}\right) f_{S}\left(\boldsymbol{p}_{4}^{*}\right)\left(1-f_{S}^{\Delta}\left(\boldsymbol{p}_{2}^{*}\right)\right)\left(1-f_{S}^{\Delta}\left(\boldsymbol{p}^{*}\right)\right) \\
& \left\{\left(\frac{f_{\pi N \Delta}}{m_{\pi}}\right)^{4} 16 \frac{1}{\left(\left(p^{*}-p_{3}^{*}\right)^{2}-m_{\pi}^{2}\right)^{2}}\right. \\
& {\left[\left(\left(p_{2}^{*} p_{4}^{*}\right)+m_{N}^{*} m_{\Delta}^{*}\right)\left(\left(p^{*} p_{3}^{*}\right)+m_{N}^{*} m_{\Delta}^{*}\right)\right.} \\
& \left.\left.\left(\frac{2}{3 m_{\Delta}^{* 2}}\left(p^{*} K\right)^{2}-\frac{2}{3} K^{2}\right)\left(\frac{2}{3 m_{\Delta}^{* 2}}\left(p_{2}^{*} K\right)^{2}-\frac{2}{3} K^{2}\right)\right]\right\}
\end{aligned}
$$

to the delta gain term and corresponds to the reaction $N N \rightarrow \Delta \Delta$.

The diagrams (f), (g), (h) in fig. 9 and the diagrams (d), (e), (f) in fig. 11 contain meson propagators between vertices located on different branches of the time contour. In order to calculate these diagrams, one has to know the correlation Green functions of the pions beside the causal and the anti-causal meson Green functions. In general, the correlation Green functions contain, in addition to the (real) off-shell parts, (imaginary) on-shell parts which give rise to an additional kinetic equation for the pions. Since we do not want to treat the production of mesons in this work, we have omitted these parts. Indeed, the density of pions is quite small in thermodynamical equilibrium of dense nuclear matter [32]. Most 
of the pions, which are observed in heavy-ion collisions result from the decay of deltas during the expansion phase. Finally, for the calculation of the diagrams (e) in fig. 9 and (c) in fig. 11 we use the quasi-static approximation [22, 31] of the pion propagator, thereby excluding again the on-shell propagation of the pions. A consistent description of pions is presented in ref. [33].

\section{References}

[1] J. Cugnon, T. Mizutani and J. Vandermeulen, Nucl. Phys. A352 (1981) 505

[2] L. W. Nordheim, Proc. Roy. Soc. A119 (1928) 689

[3] E. A. Uehling and G. E. Uhlenbeck, Phys. Rev. 43 (1933) 552

[4] B. Schürmann, W. Zwermann and R. Malfliet, Phys. Rep. 147 (1987) 1

[5] G. F. Bertsch and S. Das Gupta, Phys. Rep. 160 (1988) 189

[6] G. F. Bertsch, H. Kruse and S. Das Gupta, Phys. Rev. C29 (1984) 673

[7] H. Feldmeier, M. Schönhofen and M. Cubero, Nucl. Phys. A495 (1989) 337c

[8] C. M. Ko and Q. Li, Phys. Rev. C37 (1988) 2270

[9] B. Blättel, V. Koch, W. Cassing and U. Mosel, Phys. Rev. C38 (1988) 1767

[10] C. Grégoire, C. Remaud, F. Sébille and L. Vinet, Nucl. Phys. A465 (1987) 317

[11] Y. Ivanov, V. N. Russkikh, M. Schönhofen, M. Cubero, B. L. Friman and W. Nörenberg, Z. Phys. A 340 (1991) 385 and to be published

[12] T. Ericson and W. Weise, "Pions and Nuclei", Clarendon Press, Oxford 1988

[13] B. Serot and J. D. Walecka, Adv. Nucl. Phys. 16 (1986) 1, eds. J. W. Negele and E. Vogt, Plenum Press, New York

[14] H. Th. Elze, M. Gyulassy, D. Vasak, H. Heinz, H. Stöcker and W. Greiner, Mod. Phys. Lett. A2 (1987) 451

[15] St. Mrówczyński and U. Heinz, Regensburg preprint TPR-92-37

[16] L. P. Kadanoff and G. Baym, "Quantum Statistical Mechanics", Benjamin, New York 1962

[17] P. C. Martin and J. Schwinger, Phys. Rev. 115 (1959) 1342

[18] L. V. Keldysh, Sov. Phys. JETP 20 (1964) 1018

[19] P. Danielewicz, Ann. Phys. 152 (1984) 239 
[20] N. P. Landsman and C. G. van Weert, Phys. Rep. 145 (1987) 141

[21] G. E. Brown and W. Weise, Phys. Rep. 22 (1975) 279

[22] B. ter Haar and R. Malfliet, Phys. Rep. 149 (1987) 207

[23] D. Lurié, "Particles and Fields", John Wiley \& Sons, New York 1968

[24] C. Itzyckson and B. Zuber, "Quantum Field Theory", McGraw Hill, New York 1984

[25] E. E. van Faassen and J. A. Tjon, Phys. Rev. C28 (1983) 2354

[26] H. T. Williams, Phys. Rev. C31 (1985) 2297

[27] Landolt-Börnstein, New Series Vol. I/9a, Springer-Verlag, Berlin 1980

[28] B. ter Haar and R. Malfliet, Phys. Rev. C36 (1987) 1611

[29] M. Schönhofen, PhD thesis (TH Darmstadt 1990), GSI-report 90-16

[30] B. Waldhauser, J. A. Maruhn, H. Stöcker and W. Greiner, Phys. Rev. C38 (1988) 1003

[31] E. E. van Faassen and J. A. Tjon, Phys. Rev. C30 (1984) 285

[32] M. Schönhofen, M. Cuber^, M. Gering, M. Sambataro, H. Feldmeier and W. Nörenberg, Nucl. Phys. A504 (1989) 875

[33] M. Cubero, PhD thesis (TH Darmstadt, 1990), GSI-report-90-17;

M. Cubero, M. Schönhofen, B. Friman and W. Nörenberg, to be published 


\section{Tables:}

Table 1: Fields of the QHD model.

\begin{tabular}{|c|c|c|c|c|}
\hline field & spin & isospin & particle & mass \\
\hline \hline$\Psi_{N}$ & $\frac{1}{2}$ & $\frac{1}{2}$ & nucleon & $m_{N}$ \\
$\Psi_{\Delta}^{\mu}$ & $\frac{3}{2}$ & $\frac{3}{2}$ & delta & $m_{\Delta}$ \\
$\Phi$ & 0 & 0 & $\sigma$-meson & $m_{\sigma}$ \\
$\omega^{\mu}$ & 1 & 0 & $\omega$-meson & $m_{\omega}$ \\
$\vec{\pi}$ & 0 & 1 & pion & $m_{\pi}$ \\
\hline
\end{tabular}

Table 2: Coupling constants and cut-off parameters for describing the nucleon-nucleon cross sections in the Born approximation.

\begin{tabular}{|c|c|c|c|c|}
\hline & scalar & vector & pseudo-scalar & $\pi N \Delta$ \\
\hline$g$ & 6.60 & 8.85 & 13.45 & $1.7 / m_{\pi}$ \\
\hline$\Lambda[\mathrm{MeV}]$ & 860 & 720 & 693 & 911 \\
\hline
\end{tabular}

Table 3: Mean-field coupling constants for the two parameter sets (i) and (ii) as well as the corresponding values of the nucleon effective mass $m_{N 0}^{*}$ and the compression modulus $K_{0}$ of nuclear matter at saturation. The relative coupling strengths $B$ and $C$ are defined by $B=b / m_{N} g_{\sigma}^{3}$ and $C=c / g_{\sigma}^{4}$, respectively.

\begin{tabular}{|c|c|c|c|c|c|c|}
\hline $\begin{array}{c}\text { parameter } \\
\text { set }\end{array}$ & $g_{\sigma}$ & $g_{\omega}$ & $B$ & $C$ & $\begin{array}{c}m_{N 0}^{*} \\
{\left[m_{N}\right]}\end{array}$ & $\begin{array}{c}K_{0} \\
{[\mathrm{MeV}]}\end{array}$ \\
\hline \hline (i) & 9.57 & 11.67 & 0 & 0 & 0.56 & 540 \\
\hline (ii) & 7.93 & 6.70 & $0.17788 \times 10^{-1}$ & $0.39674 \times 10^{-1}$ & 0.85 & 210 \\
\hline
\end{tabular}




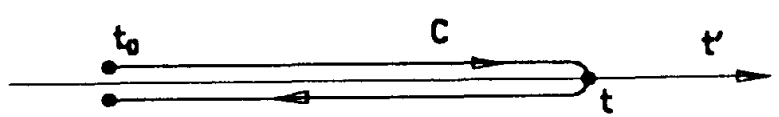

Fig. 1. Time contour $C$. 

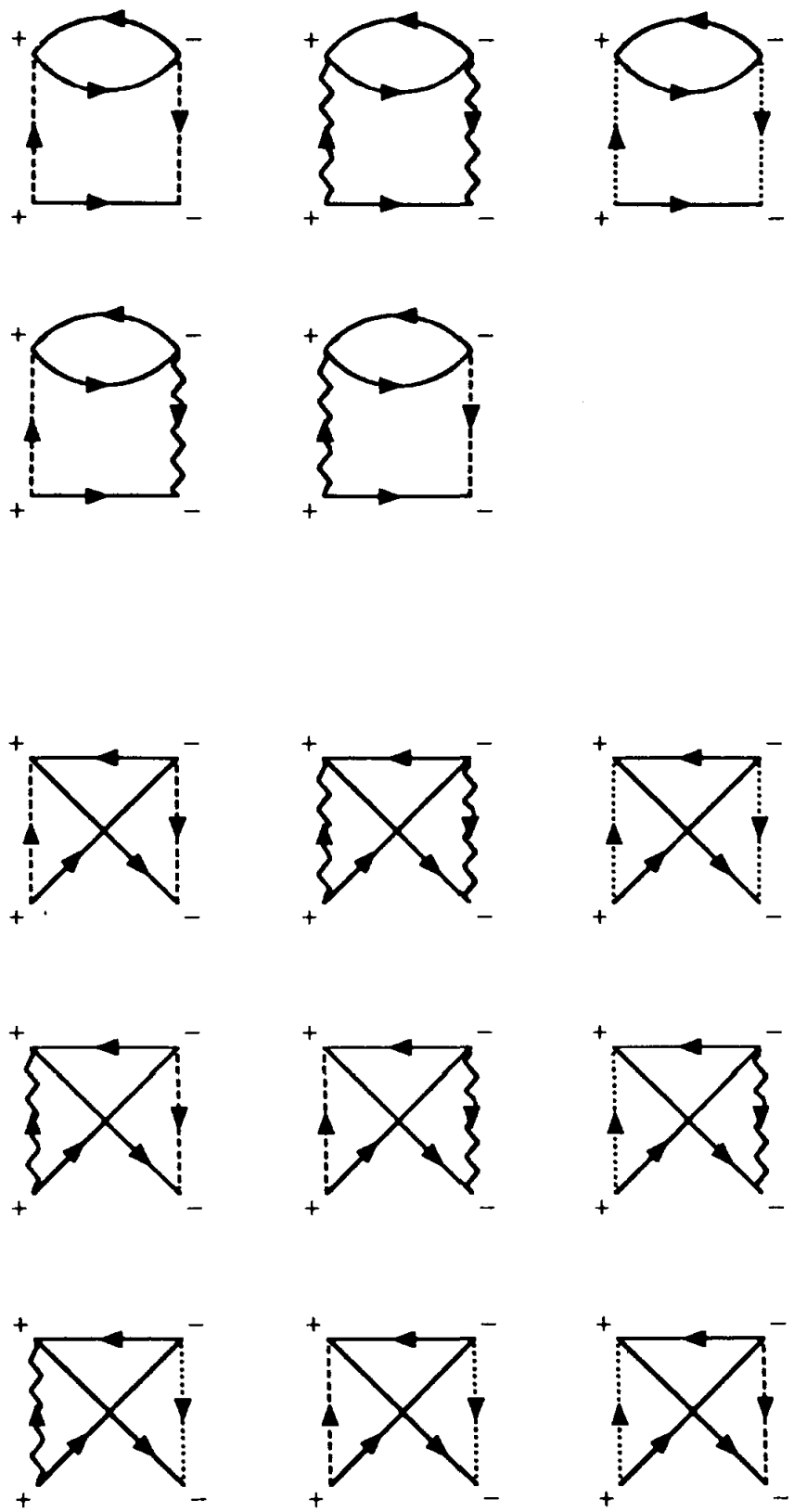

Fig. 2. Born diagrams for the elastic part of the self-energy $-i \Sigma^{<}$. The signs \pm refer to the location of the vertices on the time contour: + corresponds to the causal (upper), - to the anti-causal (lower) branch. The solid, dashed, wavy and dotted lines denote the propagators of mucleons and scalar, vector and pseudo-scalar mesons, respectivcly. 


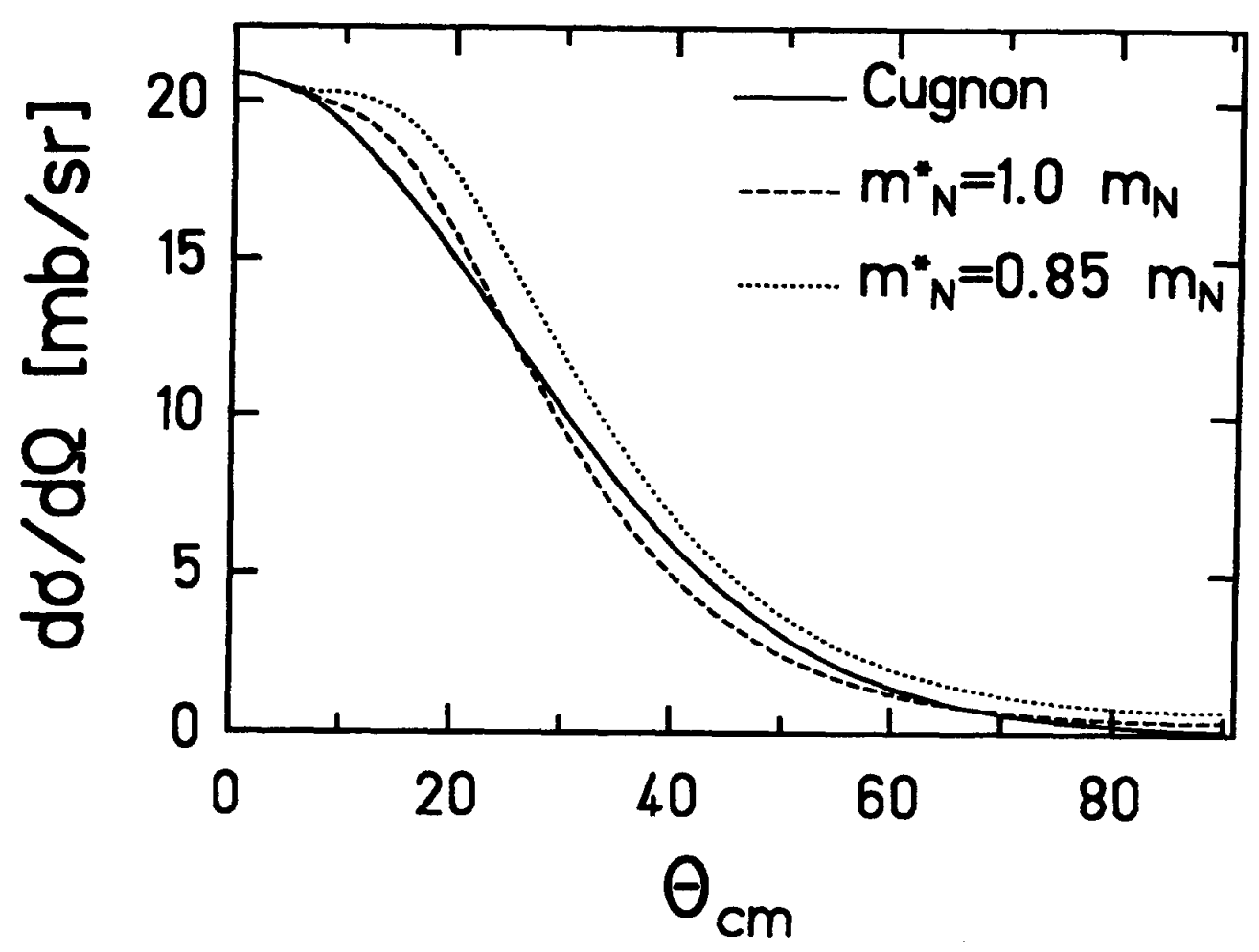

Fig. 3. Comparison of the elastic differential nucleon-nucleon cross section, calculated for $m_{N}^{*}=m_{N}$ and $m_{N}^{*}=0.85 m_{N}$, with experimental results [1] at $1 \mathrm{GeV}$. 


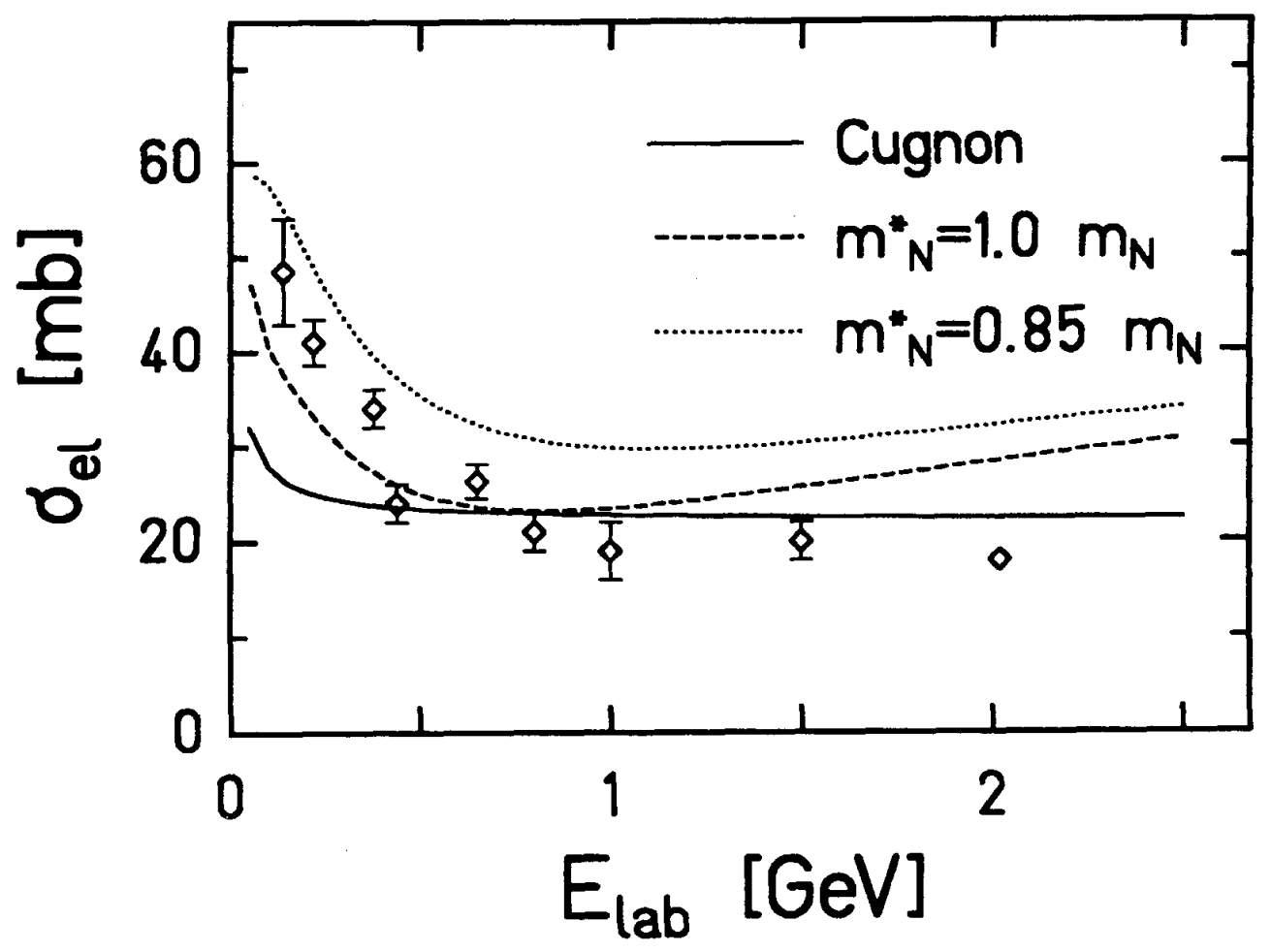

Fig. 4. Total elastic nucleon-nucleon cross section as a function of bombarding energy for $m_{N}^{*}=m_{N}$ (dashed line) and $m_{N}^{*}=0.85 m_{N}$ (dotted line). The experimental data points are from ref. [27] and the solid line gives the fit by Cugnon et al. [1]. 


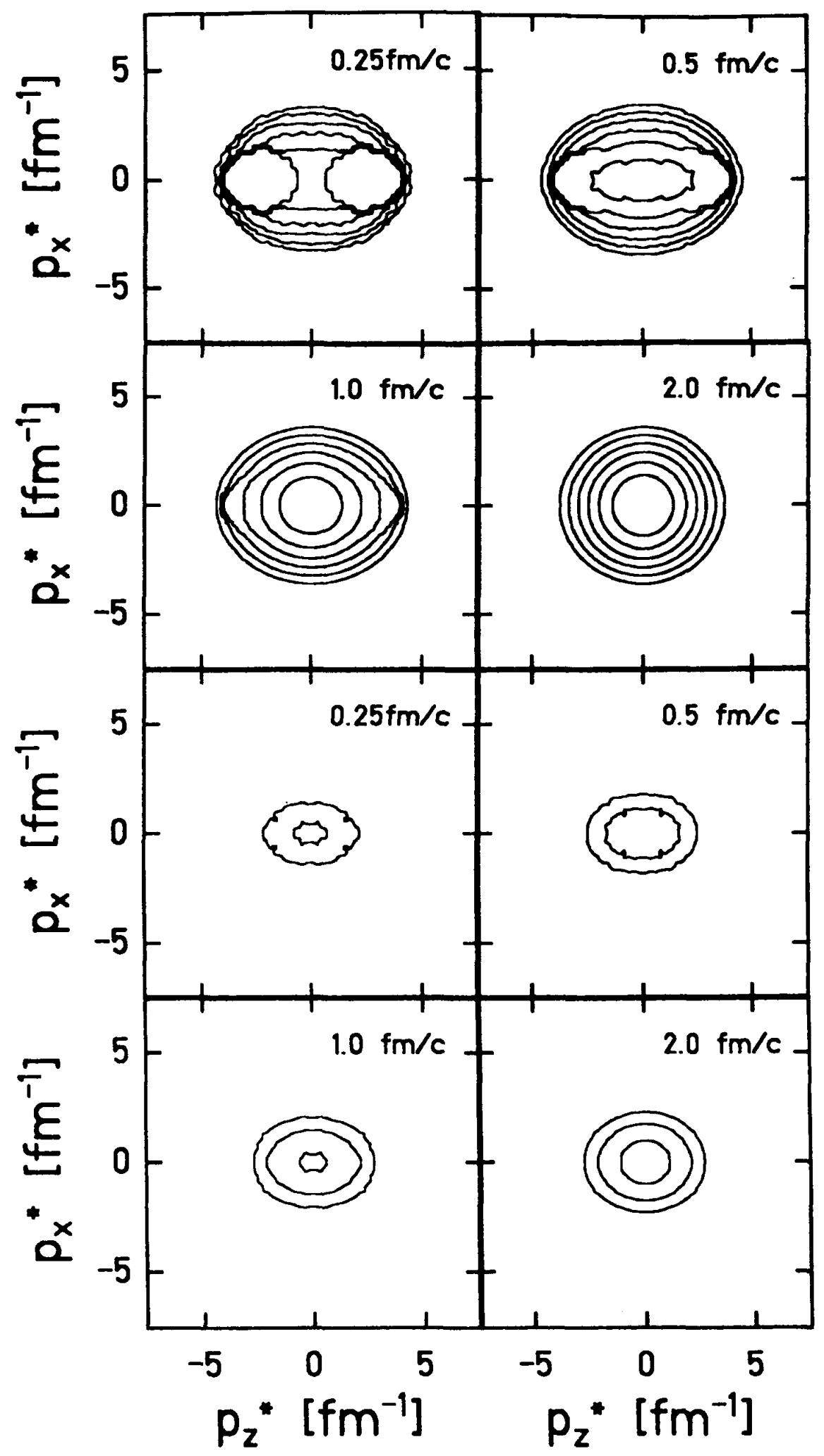

Fig. 5. Momentum distributionn of nucleons (upper part) and deltas (lower part) as function of time obtained with the mean-field Walecka model (version (i)). The contour lines indicate the values $0.01,0.02,0.04,0.08,0.16,0.32,0.64$. 


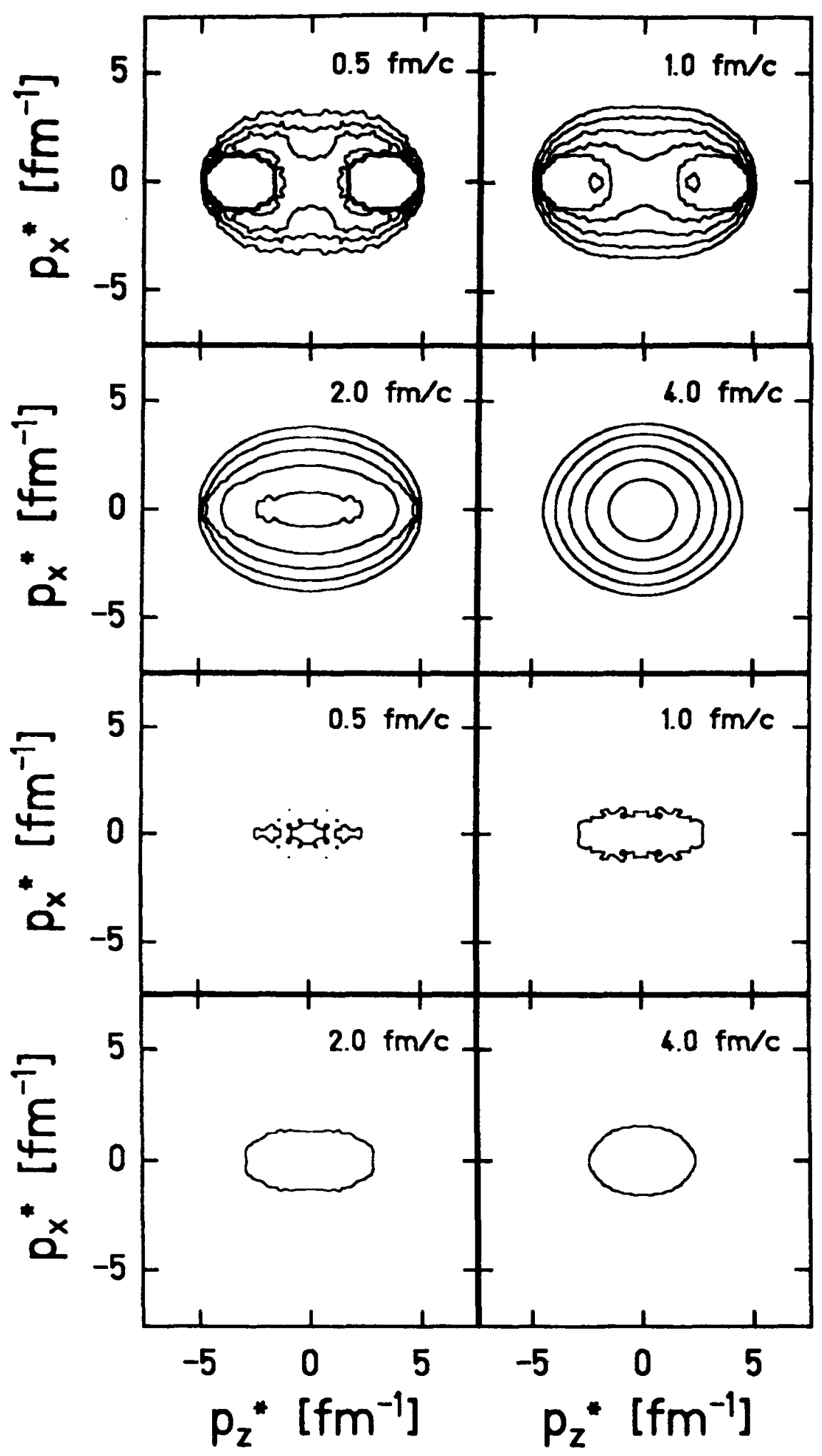

Fig. 6. Momentum distribution of nucleons (upper part) and deltas (lower part) as function of time obtained for a mean-field model with self-interactions of the $\sigma$-field (version (ii)). The contour lines indicate the values $0.01,0.02,0.04,0.08$, $0.16,0.32,0.64$. 


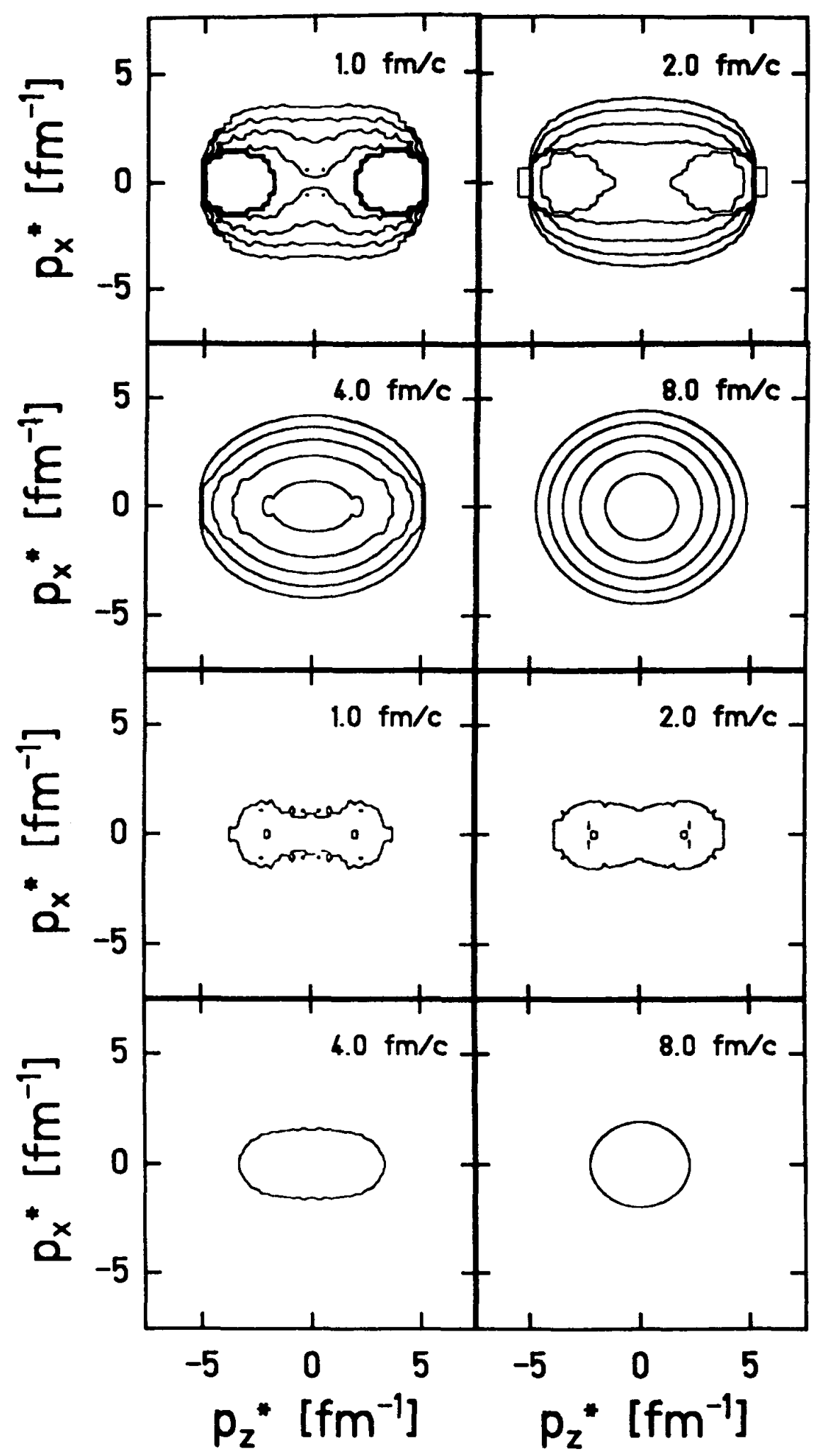

Fig. 7. Momentum distribution of nucleons (upper part) and deltas (lower part) as function of time without mean field (version (iii)). The contour lines indicate the values $0.01,0.02,0.04,0.08,0.16,0.32,0.64$. 


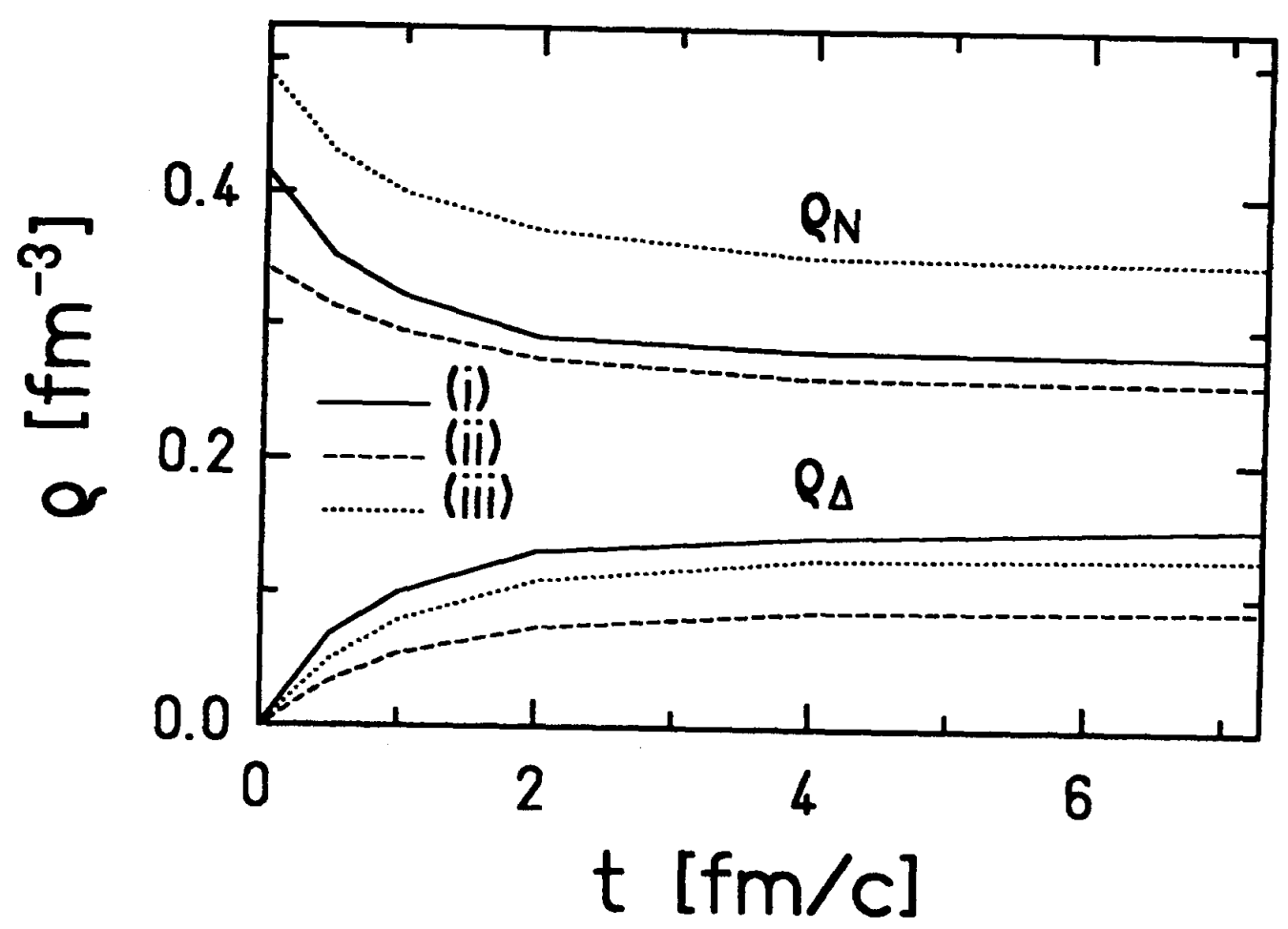

Fig. 8. Baryonic densities of nucleons and deltas as a function of time for the mean-field assumptions (i) to (iii). 
(a)

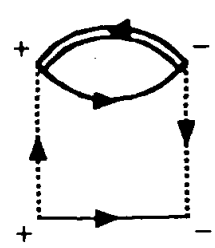

(d)

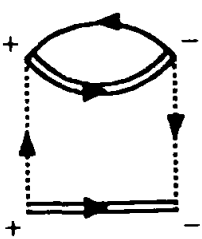

(g) (b)

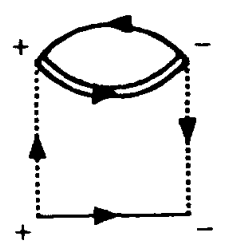

(e)

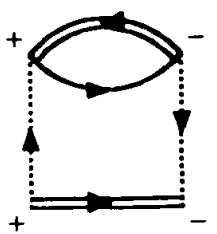

(h)

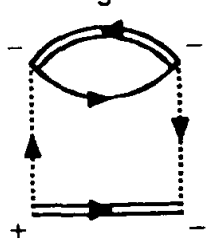

(c)

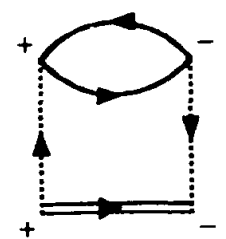

(f)

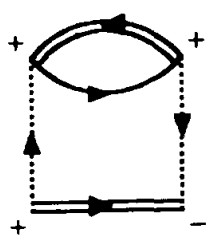

Fig. 9. Direct inelastic diagrams for $-i \Sigma^{<}$, which contribute to the kinetic equation for the nucleons.
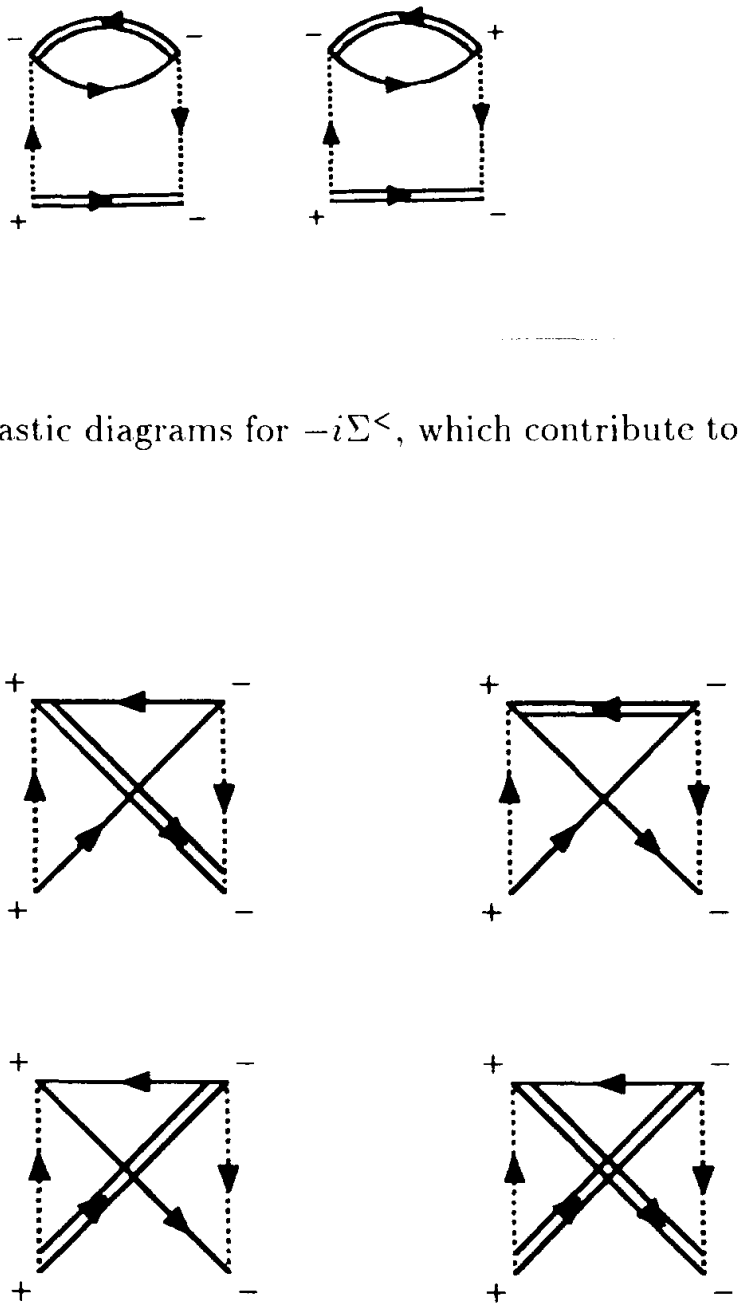

Fig. 10. Exchange diagrams for $-i \Sigma^{<}$, which contribute to the kinetic equation for the nucleons. 


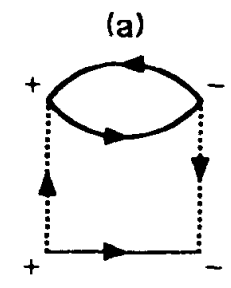

(d)

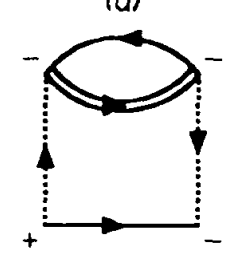

(g)

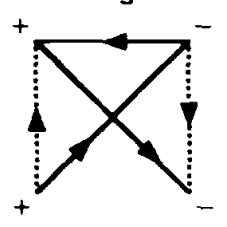

(b)

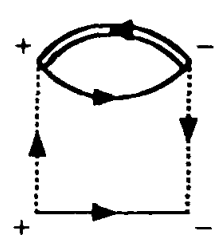

(e)

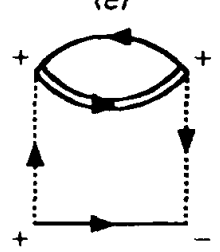

(h)

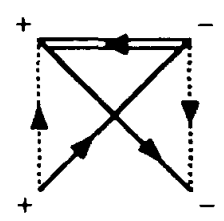

(c)

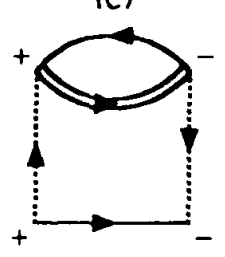

(f)

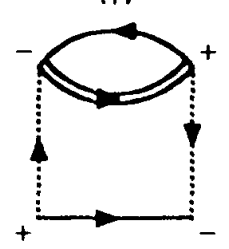

Fig. 11. Direct and exchange diagrams for $-i \Sigma^{<}$, which contribute to the kinetic equation for the deltas. 
